\title{
Ocean modeling on unstructured meshes
}

\author{
S. Danilov* \\ Alfred Wegener Institute for Polar and Marine Research, Bremerhaven, Germany
}

\begin{abstract}
Unstructured meshes are common in coastal modeling, but still rarely used for modeling the large-scale ocean circulation. Existing and new projects aim at changing this situation by proposing models enabling a regional focus (multiresolution) in global setups, without nesting and open boundaries. Among them, finite-volume models using the C-grid discretization on Voronoi-centroidal meshes or cell-vertex quasi-B-grid discretization on triangular meshes work well and offer the multiresolution functionality at a price of being 2 to 4 times slower per degree of freedom than structured-mesh models. This is already sufficient for many practical tasks and will be further improved as the number of vertical layers is increased. Approaches based on the finite-element method, both used or proposed, are as a rule slower at present. Most of staggered discretizations on triangular or Voronoi meshes allow spurious modes which are difficult to filter on unstructured meshes. The ongoing research seeks how to handle them and explores new approaches where such modes are absent. Issues of numerical efficiency and accurate transport schemes are still important, and the question on parameterizations for multiresolution meshes is hardly explored at all. The review summarizes recent developments the main practical result of which is the emergence of multiresolution models for simulating large-scale ocean circulation.

Key words: Unstructured meshes, Finite-volume and finite-element methods, large-scale ocean circulation modeling
\end{abstract}

\section{Introduction}

Over the last decade the ocean circulation modeling on unstructured meshes was a subject of ongoing research, as partly highlighted in reviews by Pain et al. (2005) and Piggott et al. (2008). A number of new models has

${ }^{*}$ Corresponding author.

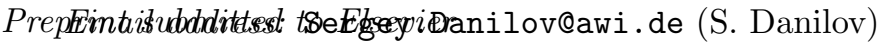

May 8, 2013 
been announced, such as FVCOM (Chen et al. (2003)), ICOM/Fluidity (Ford et al. (2004) and Piggott et al. (2008)), FESOM (Danilov et al. (2004) and Wang et al. (2008)), SLIM (White et al. (2008a), Blaise et al. (2010) and Kärnä et al. (2013)), the model by Stuhne and Peltier (2006), SUNTANS (Fringer et al. (2006)), MIKE 21 \& MIKE 3 Flow Model FM (http://www.mikebydhi.com), ELCIRC (Zhang et al. (2004)) or SELFE (Zhang and Baptista (2008)). There are older, largely coastal or estuarine modeling efforts, such as ADCIRC (Westerink et al. (1992)), QUODDY (Lynch et al. (1996)), TELEMAC (Hervouet (2000) and Hervouet (2007)) or UnTRIM (Casulli and Walters (2000)). Two new projects with focus on large-scale atmosphere and ocean circulation, MPAS (http://mpas.sourceforge. net/) and ICON (www.mpimet.mpg.de/en/science/ models/icon.html), also include ocean components. The numerical principles of MPAS approach are described by Thuburn et al. (2009) and Ringler et al. (2010), and the first results of MPAS-ocean simulations are very encouraging (Ringler et al. (2013). There are many more models either designed for hydrology tasks or focused solely on barotropic shallow water which are not listed here.

Unstructured meshes suggest flexibility with respect to resolving the geometry of basins. By locally refining computational meshes they also enable one to simulate regional dynamics on a global mesh with an otherwise coarse resolution. The geometrical aspect is of utmost importance for coastal applications where computational domains involve complex-shaped coastlines and very different scales, from basin size to details of river estuaries or riverbeds. Additionally, by locally scaling the meshes as $H^{1 / 2}$ or $H /|\nabla H|$, where $H$ is the water depth, one can take care of the variable surface wave speed or rapidly changing bottom topography, respectively, optimizing the mesh for simulations of tidally driven flows. The dynamical aspect is rather of interest for large-scale ocean modeling, as it offers an effective nesting approach in a global configuration free of open boundaries. The purely geometrical motivation is relevant too, but its focus shifts to places like straits, overflows or the continental break.

The research community dealing with unstructured meshes aims at providing a platform for multiresolution ocean modeling. Numerous coastal studies performed with FVCOM or ADCIRC (see their web sites for the lists of publications) vividly illustrate that the span of resolved scales can be very large (in excess of two orders of magnitude). And yet, further direct expansion from coastal toward large scales can be unpractical because the 
spectrum of temporal and spatial scales becomes too wide. Indeed, the mere equilibration on the global scale may take tens (if not hundreds) of years, and the fine-resolved coastal part will become an unnecessary burden. Similarly, although large-scale ocean simulations on global meshes with the refinement factor of about 30-50 have already been reported (see, e. g., Wang et al. (2009)), it seems unlikely that this factor will be increased much further without additional measures. Given the coarse resolution of $50-100 \mathrm{~km}$, such a refinement is already sufficient to reach a kilometer scale. Going beyond it may imply new physics (e. g, non-hydrostatic effects) or prohibitively large CPU cost because the time step is determined by the smallest size.

It is thus unlikely that unstructured meshes will offer a solution suited to simulate across all scales simultaneously while fully abandoning nesting. Considerations of numerical efficiency, let alone the difference in dynamics, parameterizations and mesh design, indicate that some separation between coastal and large-scale applications is likely to be preserved. This separation notwithstanding, the refinement already used in practice on unstructured meshes by far exceeds that of traditional nesting, which warrants the place for unstructured-mesh models as bridging the gap between scales and reducing the need in nesting to minimum.

Given the number of existing efforts and promises made, it seems timely to briefly summarize the achievements, questions and difficulties and draw conclusions on the further development. We do not aim at full account, leaving aside such 'high-tech' perspectives as mesh adaptivity. Instead, we try to explain what are the main difficulties as compared to structured meshes, what is already possible in practice and what should be improved, using the models known to us as an illustrating material. Our experience and hence conclusions are biased to the large-scale modeling, which is less forgiving to numerical errors than the coastal one simply because of much longer time scales. The importance of geostrophic adjustment and balance in the largescale dynamics is the other distinguishing feature of large-scale modeling.

Speaking broadly, the main difficulty faced by models formulated on unstructured meshes lies in spurious modes maintained by discretizations. While certain spurious modes are known to occur even on regular finitedifference grids (like pressure modes on A and B grids or inertial modes on C-D grids), handling them on unstuctured meshes is more difficult. Most of staggered discretizations support branches of spurious modes which can be excited by nonlinear dynamics. Additionally, unstructured-mesh models are more expensive per degree of freedom. 
Because of relatively short integration time, coastal models formulated on unsructured meshes are less vulnerable to spurious modes or to errors occurring from stabilizing them. More importantly, they offer a geometric flexibility which is difficult to achieve by other means. As a result, most of unstructured-mesh models are coastal (with ADCIRC, FVCOM, UnTRIM, SELFE and others having a long record of successful applications). The research here only seeks how to improve their already good performance or works on new functionality (like nonhydrostatic and ice components in FVCOM).

The need to handle spurious modes and the higher computational cost explain why the attempts to large-scale modeling on unstructured meshes have not always been successful or are taking too long. Unstructured-mesh large-scale ocean models now include FESOM and MPAS, with ICON working to the goal and other projects (SLIM, ICOM and FVCOM) considering it. The understanding available now is already sufficient to propose solutions that are good enough to be used in practice. However, examples showing the utility of the approach are only beginning to appear.

For convenience, section 2 schematically explains main discretization methods used on unstructured meshes. It can safely be omitted if the reader is familiar with them. The following sections discuss the vertical coordinate, main discretization types and their properties, conservation properties, advection schemes, and reiterate on practical examples. The final sections present discussions and conclusions.

\section{Main approaches}

In order to facilitate further reading this section briefly sketches basic technologies of writing discretized equations on unstructured meshes the finite element $(\mathrm{FE})$ and finite volume (FV) methods. Within the FE method one distinguishes between continuous and discontinuous representations. Sometimes one uses the notion of mimetic differencing (or mimetic approach), which is related to both $\mathrm{FE}$ and $\mathrm{FV}$ methods or their combination, and places focus on mimicking the properties of continuous operators. Regular courses like Zienkiewicz and Taylor (2000), Blazek (2001) or Li (2006) contain many details.

We select an advection-diffusion equation for a tracer $T$ to illustrate the basic approaches,

$$
\partial_{t} T+\nabla \cdot\left(\mathbf{u} T-K_{h} \nabla T\right)+\partial_{z}\left(w T-K_{v} \partial_{z} T\right)=0,
$$


with $\nabla=\left(\partial_{x}, \partial_{y}\right)$ and boundary condition that tracer flux is equal to $Q$ at the upper surface while other surfaces are 'insulated'. Here $\mathbf{u}$ and $w$ are, respectively, the horizontal and vertical components of advecting velocity, and $K_{h}$ and $K_{v}$, the diffusivity coefficients. For definiteness assume that the computational mesh is vertically extruded from a triangular surface mesh. The vertical prisms are cut into smaller prisms by a set of $z$-surfaces.

\subsection{Continuous finite elements}

According to the FE method, all fields are expanded in test functions defined on the elements of an unstructured mesh and belonging to an appropriate functional space. We will not touch on the details of spaces here. In the simplest case the test functions are polynomials of low order with support limited to one (usually discontinuous) or several neighboring elements (prisms). The discretized equations are obtained by projecting dynamic equations on a set of test functions. They frequently coincide with the basis functions, giving the so-called Galerkin projection. Upwind-biased test functions lead to the Petrov-Galerkin method. By its idea, the FE method resembles the spectral method.

Expand $T$ in a set of basis functions $N_{j}=X_{j}(x, y) Z_{j}(z)$ defined on prismatic elements, $T=T_{j}(t) N_{j}$ (summation over repeating indices is implied if $T_{j}$ is involved). Depending on the choice of functions, the index $j$ can list mesh elements or vertices (nodes) or additional nodes in elements or on their faces. A simple example is the continuous $P_{1}$ representation $(P$ stands for polynomial, and 1 for its degree; see section 4 for more examples). In this case $X_{j}$ and $Z_{j}$ equal 1 at vertex $j$ and go linearly to zero at neighboring horizontal and vertical vertices respectively, so that $T=T_{j}(t) N_{j}$ is a bilinear interpolation which is continuous across the faces. If prisms are split into tetrahedra, the 3D linear representation becomes possible, $N_{j}=N_{j}(x, y, z)$, and the expansion $T_{j} N_{j}$ implies a linear interpolation in three dimensions.

Next, equation (1) is re-written in a weak form as

$$
\int\left(M_{i} \partial_{t} T-\mathbf{F}_{h} \nabla M_{i}-F_{v} \partial_{z} M_{i}\right) d \Omega=\int Q M_{i} d S
$$

where $M_{i}$ is an appropriate test function, $\mathbf{F}_{h}$ and $F_{v}$ are the horizontal and vertical components of fluxes and integration by parts has been performed. If $M_{i}=N_{i}$, one arrives at the Galerkin discretization

$$
M_{i j} \partial_{t} T_{j}+\left(A_{i j}+D_{i j}^{h}+D_{i j}^{v}\right) T_{j}=S_{i}
$$


where $M_{i j}=\int N_{i} N_{j} d \Omega, A_{i j}=-\int N_{j}\left(\mathbf{u} \cdot \nabla N_{i}+w \partial_{z} N_{i}\right) d \Omega, D_{i j}^{h}=-\int K_{h}\left(\nabla N_{i}\right)\left(\nabla N_{j}\right) d \Omega$ and $\left.D_{i j}^{v}=-\int K_{v} \partial_{z} N_{i} \partial_{z} N_{j}\right) d \Omega$ are, respectively, mass, advection, horizontal and vertical diffusion matrices, and $S_{i}=\int N_{i} Q d S$ is the source term. Note that (2) requires that $N_{i}$ are at least continuous (derivatives have to be bounded). The approach implemented in (3) will be referred to as the continuous Galerkin (CG) discretization.

Modifications are needed to the approach above on prismatic meshes if the level surfaces deviate from the $z$-coordinate. In this case functions $N_{j}$ are specified on so-called standard (parent) elements (unit height rectangular prisms with the base formed by a unit rectangular triangle), and coordinate transforms from the physical space to the parent space are performed in integrals for matrix elements. For linear tetrahedral elements the modification is trivial since the Jacobians of transforms are elementwise constant. They are coordinate dependent in a general case and quadrature rules of appropriate order are needed to perform computations.

There are several immediate implications. First, in contrast to finitedifference codes, time derivatives in (3) are coupled through mass matrices ( $M_{i j}$ above) which are usually non-diagonal and global for the CG discretization (for example, on triangular prismatic meshes row $i$ of $M_{i j}$ will contain about 20 non-zero entries for linear functions). Keeping them improves accuracy by reducing numerical dispersion in advection schemes (see, e. g., Donea and Huerta (2003)), but iterative solvers must then be used. Diagonal, or lumped, approximations are sometimes selected to reduce the incurring computational burden, yet with an adverse effect on the accuracy of advection. According to Le Roux et al. (2009), lumping has a moderate (yet negative) effect on the dispersion properties of resolved waves, but this has been tested only for several FE pairs.

Second, the implicit treatment of vertical diffusion, needed as a rule by ocean circulation models, implies inversion of global matrices too, this time because of horizontal connections in $D^{v}$. These connections create even larger numerical difficulties in hydrostatic codes, making hydrostatic balance or continuity equation difficult to solve for pressure and vertical velocity respectively.

Third, since test functions satisfy $\sum_{i} M_{i}=1$ (partition of unity), global tracer conservation is immediately recovered by summing over $i$. Local conservation is the equation itself, but it does not take the flux form a user is inclined to have. Computing 'common sense' transports (like the meridional overturning) entails uncertainties (see discussion by Sidorenko et al. (2009)). 
These issues is the reason why the CG FE method is not optimal for ocean modeling, as will be explained further in more detail.

\subsection{Finite-volumes}

The FV method derives discretized equations by introducing control volumes and integrating over them. We consider the simplest case when the control volumes coincide with prisms the mesh is composed of (see section 4 for more variants). The equations of motion are integrated over the control volumes and their flux divergence terms are expressed, via the Gauss theorem, as fluxes out of the control volumes. Due to this strategy, local and global balances are ensured on the discrete level. To illustrate the FV method, it is applied to equation (1). Integrating (1) over prism $(n, i)$ located in layer $n$ below triangle $i$ one obtains

$$
\partial_{t} \int T d \Omega_{n i}+\sum_{k=1}^{3}\left(\mathbf{F}_{h} \cdot \mathbf{n} S\right)_{n k}+\left(F_{v} S\right)_{n i}-\left(F_{v} S\right)_{(n+1) i}=0,
$$

with $\left(F_{v} S\right)_{1 i}=Q_{i} S_{i}$. Here $k$ enumerates the edges of triangle $i, \mathbf{n}$ is the outer normal on vertical faces, $S$ the area of faces and $S_{i}$ is the area of surface triangle $i$. The discrete tracer values are introduced as $T_{n i}=\int T d \Omega_{n i} / V_{n i}$, where $V_{n i}=S_{i} h_{n}$ is the volume of prism $(n, i)$ and $h_{n}$ the layer thickness (the prism height). The essence of the FV approach lies in estimating the fluxes leaving the control volume in terms of $T_{n i}$ and volume-mean values at neighboring control volumes. Generally, reconstruction of fields or their gradients is performed to accurately assess the fluxes. The estimates are discontinuous across the face and are replaced with 'numerical' fluxes. Obvious examples are furnished by centered or upwind fluxes, and they are frequently limited to warrant monotonicity. Linear field reconstructions are formally sufficient for the second order convergence. They can be easily implemented as they only require the information from the nearest neighbors. They are, however, not always sufficient for oceanic applications, calling for higher-order or gradient reconstructions.

On the conceptual level, the procedure is similar to that of structuredmesh FV codes such as MITgcm (Marshall et al. (1997)). The mesh unstructuredness, however, makes reconstructions and limiting less straightforward and involves noticeably higher computational effort.

Note that in contrast to CG FE no horizontal connections are introduced for vertical derivatives. This makes FV approach better suited for hydrostatic codes. 


\subsection{Discontinuous FE}

Discontinuous finite elements can be considered as a generalization of both FV and CG FE approaches. One gets a weak formulation by integrating over elements interiors with some appropriate test function $M$ and requiring the result to hold for all $M$ from some functional space. In this case the result is

$$
\sum_{n i}\left(\int\left(M \partial_{t} T-\mathbf{F}_{h} \nabla M-F_{v} \partial_{z} M\right) d \Omega_{n i}-\int Q M d S_{1 i} \delta_{1 n}+\int M \mathbf{F} \mathbf{n} d S_{i n}\right)=0,
$$

where $n$ and $i$ number the elements in vertical and horizontal directions, and integration in the last term is over the surface of element. The (polynomial) representation for $T$ is restricted to element interiors, and is discontinuous across the elemental boundaries. Because of this, the elements are disconnected and (5) is incomplete unless certain continuity penalties are added to the weak formulation. More commonly, the fluxes $\mathbf{F}$ are considered to be the 'numerical' fluxes. They provide the only way the elements are connected. They combine flux estimates from elements sharing the face with relevant continuity constraints to ensure accuracy and stability. A simple example is the upwind estimate when the flux is taken as a boundary limit on the respective upwind element (additional constrains are still necessary to properly tackle the diffusion terms).

As compared to the FV method, the high-order polynomials of the discontinuous Galerkin (DG) FE method spare the need of reconstructions. As compared to continuous elements, mass matrices now connect only local degrees of freedom (DOF) inside elements, which makes their direct inversion feasible. This is, however, achieved through a noticeably increased number of degrees of freedom inside elements. Because of incurring computational burden practical applications of discontinuous elements in ocean modeling are rare (see, e.g. Dawson et al. (2006), Blaise et al. (2010), Comblen et al. (2010), Kärnä et al. (2013)).

\subsection{Mimetic approach}

A general approach to unstructured polygonal meshes, combining useful sides of FV and FD methods, came under the name of mimetic finite difference. Mimetic discretization methods create discrete versions of partial differential operators that are exact in some sense, or mimic (hence the name) the properties of continuous operators. These, for example, include the requirement that the discrete operators of divergence and gradient are 
negative adjoint of each other in the energy norm, as well as the requirements that $\nabla \times \nabla T=0$ or $\nabla \times(\mathbf{k} \times \mathbf{u})=-\nabla \cdot \mathbf{u}$ hold on the discrete level, where $\mathbf{k}$ and $\mathbf{u}$ are, respectively, a unit vertical vector and the horizontal velocity, which is needed to obtain the discrete vorticity balance from discretized momentum equations. Certain FV and FE discretizations are mimetic, but many implementations used in ocean modeling are not. The symmetry between gradient and divergence is achieved by selecting an appropriate scalar product and defining one operator as the negative adjoint of the other one, which is automatically the case for CG FE. The maintenance of (potential) vorticity and enstrophy balances depends on how the discrete vorticity is defined and cannot be achieved in many cases.

While the topic has a long history, in the context of atmospheric modeling it in fact appears already in Arakawa's works (see Arakawa (1966), Arakawa and Lamb (1981)) dealing with the maintainance of energy and enstrophy balance on C-grids. Of current model development efforts known to the author the C-grid based approach used by MPAS (as described by Ringler et al. (2010)) and the ICON-ocean (P. Korn, private communication) are mimetic. The quasi-B-grid (cell-vertex) approach described in Danilov (2012) can be made mimetic too. Cotter and Shipton (2012) introduce the families of mixed finite elements that satisfy conditions of finite element exterior calculus with build-in mimetic properties, and Cotter and Thuburn (2012) offer a more theoretical introduction to the topic. There is vast literature on mimetic differencing outside the atmospheric/ocean modeling (see, e.g., Hyman and Shashkov (1997), Subramanian and Perot (2006) and references therein).

\section{Unstructured meshes and the vertical coordinate}

\subsection{Vertical coordinate}

Unstructured meshes do not offer new solutions for the vertical representation as compared to regular meshes. For one thing, nodes must be vertically aligned to facilitate computations of hydrostatic pressure and minimize aliasing of horizontal pressure gradients by the vertical one. The ICOM group was exploring the possibility of fully $3 \mathrm{D}$ unstructured meshes, motivated by the task of 3D mesh adaptivity. Although feasible in principle (Kramer et al. (2010)), this approach encounters difficulties in solving for pressure in situations relevant for ocean large-scale dynamics. Assuming the vertical alignment, the 'unstructuredness' relates only to the surface mesh. The surface mesh defines prisms which are further cut into smaller prisms by 
layer surfaces. These can be geopotential, terrain-following, isopycnal or any their combination, same as in finite-difference models. In finite-element (FE) codes a further subdivision step is sometimes made: each mesh prism is split into three tetrahedra (FESOM, ICOM). 'Partial' or 'shaved' cells and also the $z^{*}$ coordinate are possible in FV codes. In all cases the ALE (arbitrary Lagrangian Eulerian) approach can easily be applied (see Donea and Huerta (2003) for general exposition, and White et al. (2008b) and Ringler et al. (2013) for FE and FV applications, respectively).

Still, the unstructuredness opens some new perspectives. First, the surface triangular mesh can be generated so that it includes certain discretized isobaths corresponding to the level surfaces. In that case one can get smooth bottom representation on $z$-coordinate grids if shaved cells are used. There will be improvement even with full cells because many local steps will be avoided. In practical terms, however, this approach can only be used in regional configurations (see Wang et al. (2008) for illustration). On global scale the continental margin represents an obvious difficulty unless one can afford resolution on a kilometer scale, yet certain alignment of mesh and topography is feasible. Much in the same vein, on terrain following meshes one can locally increase the horizontal resolution over the steep parts of the bottom. This makes the hydrostatic consistency requirement less demanding.

Second, one can easily combine terrain-following levels above some isobath and $z$-coordinate below it. The unstructured character of mesh assists in doing it seamlessly. Such functionality is suggested by SELFE (Zhang and Baptista (2008)) and FESOM (Wang et al. (2008)).

Third, many FE unstructured-mesh models assume some polynomial (e.g., piecewise-linear) representation for fields not only in the horizontal, but also in vertical direction, as is the case with SELFE, FESOM, SLIM. In that case the horizontal partial derivatives at constant $z$ are known on elements and the code may work on meshes with generalized vertical levels without the need of transforming to the new vertical coordinate. This is the approach of SELFE, SLIM, FESOM. All what is required is an appropriately constructed mesh, the code remains without changes. Clearly, the horizontal gradients can still be aliased by the vertical ones on elements with vertices at more than two levels, leading, among others, to pressure gradient errors. For this reason these models apply algorithms minimizing pressure gradient errors by default. Among the models mentioned above, FVCOM, TELEMAC and ADCIRC do transform to the terrain following vertical coordinate, UnTRIM, SUNTANS and the model by Stuhne and Peltier (2006) are formulated on 
$z$-coordinate meshes, and other models allow both approaches.

Noteworthy, the bottom may contain elements with acute angles pointing into the land or ocean on 'full-cell' $z$-coordinate meshes based on surface triangulation. They should be avoided, with implication that some trimming of the bottom is frequently required.

\subsection{Surface unstructured meshes}

A review by Greenberg et al. (2007) discusses numerous aspects of unstructured mesh design, which will not be repeated here. Goals pursued by coastal and large-scale modelers are different, and so are typical meshes used by them. Figure 1 illustrates schematically the difference in approaches. In coastal tasks dynamics are tidally dominated, and mesh is refined in shallow areas according to the speed of long gravity waves (left panel). Shallow areas are of less interest for large-scale simulations and the mesh is refined in areas where dynamics are of particular significance (right panel). Web site of FVCOM offers numerous examples of coastal meshes and related simulations, and Wang et al. (2012), Hellmer et al. (2012) and Wekerle et al. (2013) give examples of studies performed with FESOM on meshes with focus on Arctic Ocean, Antarctic Ice Shelf and Canadian Arctic Archipelago respectively.
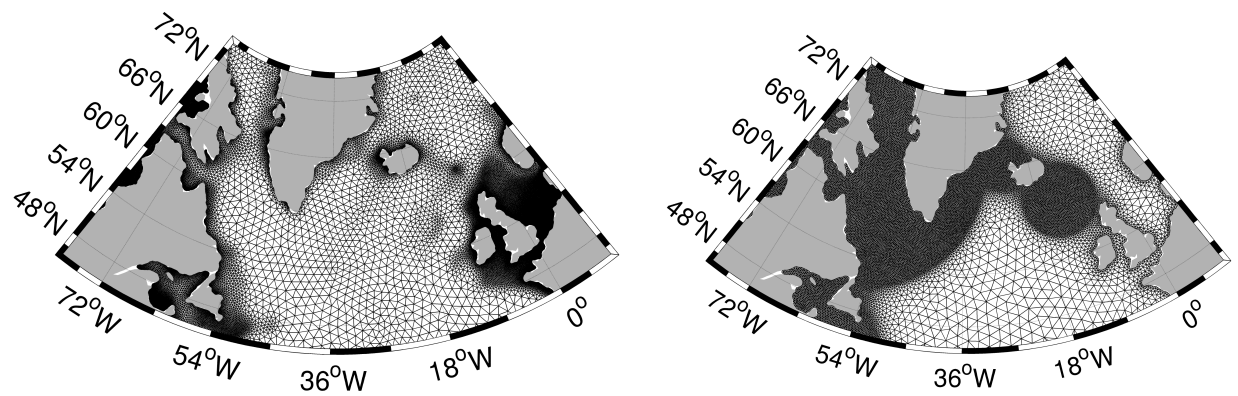

Figure 1: Mesh design for coastal (left) and global (right) simulations. In the first case the element size follows the phase speed of long surface gravity waves, but this can be overridden by geometrical requirements at the coast, in estuaries or in the vicinity of topography. In the second case the zeroth-order approximation is simply the refinement in area where dynamics are studied. Other refinements may be necessary too (not shown).

The notions of the Voronoi diagram (tessellation) and Delaunay triangulation are frequently invoked with respect to unstructured meshes. For a finite set of points $\left\{p_{n}\right\}$ in the Euclidian plane the Voronoi cell $V_{k}$ corresponding to point $p_{k}$ consist of points whose distance to $p_{k}$ is less than or equal to 
the distance to other points. It is obtained from intersection of lines equidistant to neighboring points and presents a convex polygon. Its vertices are called Voronoi vertices. The Delaunay triangulation is dual to the Voronoi diagram and is obtained by connecting triples of points $p_{k}$ associated to a Voronoi vertex. This vertex is the circumcenter for such a triangle. It has the property that there is no other point within the circumscribed circle, which helps to reduce the occurrence of triangles with small angles. The relation between the Voronoi tessellation and Delaunay triangulation is illustrated in the right panel of Fig. 1, where the dark squares are the Voronoi vertices. Generalization to spherical geometry is straightforward.

Most popular type of surface tessellation is via a Delaunay triangulation and models mentioned above use it. Triangular elements enable smooth representation of coastlines in a fairly straightforward way. There are numerous triangular mesh generators, both free and commercial, and we mention here GMSH (Lambrechts et al. (2008)), the simple generator by Persson and Strang (2004) and its more advanced implementation ADMESH (Conroy et al. (2012)) by the way of example. Depending on applications and discretization algorithms, models have different requirements to mesh quality and smoothness (resolution change rate). For example, models like UnTRIM and SUNTANS require the so-called orthogonal meshes where circumcenters are inside respective elements, which is sometimes too restrictive in complex geometries.

Local mesh nonuniformity and anisotropy may increase residual errors in the representation of operators in a general case on static meshes (but adapting meshes can benefit from stretching in along-flow direction). Ideally, mesh triangles should be as close to equilateral as possible. Local mesh quality can essentially be improved by slightly displacing the nodes and retriangulating the mesh, for example, following the procedure of Persson and Strang (2004). Mesh resolution is assigned as a rule in terms of density function. However, it is rather difficult to foresee all needed features, let alone the difference in requirements for coastal and large-scale applications. In practical terms it means that no generator suits modeler's needs $100 \%$ and in all cases multiple trials are required.

Triangles are most widely, but not solely, used elements. The early version of ICOM was formulated on an (unstructured) surface quadrilateral mesh, and the current MPAS effort is build on unstructured Voronoi meshes. A simple iterative procedure (Ringler et al. (2008)) in this case allows constructing elements in which centroids and generating points coincide (a 
centroidal Voronoi tessellation) while the size of elements follows some goal function. It leads to quasi-hexagonal meshes. Quadrilateral elements have to be strongly deformed in complex geometries to fit boundaries or refine the resolution, and with purely hexagonal elements the boundary is always castellated (but smooth coastline can be recovered by allowing pentagons).

Many models formulated with finite-volume (FV) method (e. g. FVCOM or UnTRIM) can in principle be generalized to work on meshes composed of different polygons (see illustration in Casulli and Walters (2000)), but we are only aware of coastal applications of UnTRIM that use such an approach.

This direction seems to be promising, as the meshes composed of, for example, triangles in transition zones and quasi-regular quadrilaterals in fine parts may allow substantial improvement in the quality of local advection schemes by relatively simple means. Yet it remains to see whether it will be matching the expectations in practice.

Strong inhomogeneity in the mesh resolution may cause undesirable effects like wave reflections (see, e.g., cautions expressed in Griffies et al. (2000)). Should it happen, it would imply that the mesh smoothness is inappropriate for the problem under study. Unstructured meshes do not offer miracles - one has to ensure first and foremost that residuals in representation of differential operators remain sufficiently small. Rigorous studies of possible effects of inhomogeneity in ocean context are lacking thus far. We note, however, that error analyses routinely applied with adaptive meshes can prove valuable in this context. We also note that dissipative operators are commonly scaled with resolution, so that one always tries to rather damp than reflect or scatter the perturbations.

\section{Main discretization types and their properties}

Historically, the development of unstructured-mesh ocean models was driven by coastal oceanography tasks, and was initially based on the FE method. FV codes started to appear later, and large-scale applications followed even later. The development in most cases was dictated by practical tasks while theoretical understanding was lacking. The situation is much improved now and properties of numerous discretizations are well studied. The goal here is to briefly mention existing approaches, and sketch a general picture. The preference is given to low-order discretizations. Only their horizontal part is discussed as most important. 
We note that the order of spatial convergence depends on the selected discretization. In the FE case, one expects to have the second order for linear fields, and the first order for element-wise constant fields. For the FV method, linear reconstructions are expected to provide the second order. Superconvergence with respect to particular wave propagation tasks (Bernard et al. (2008)), and reduced convergence rate on nonuniform meshes (Hanert et al. (2009), Bernard et al. (2009)) can sometimes be observed.

\subsection{Placement of variables}

Figure 2 illustrates the horizontal placement of variables on some loworder finite elements, with arrow indicating the position of normal velocities. Figure 3 introduces finite-volume discretizations, captions to figures explain the details. Below the discretizations will be listed in pairs, first the representation for velocity and then for scalar variables (elevation, pressure, temperature and salinity).

\subsubsection{Finite-elements}

Continuous $P_{1}-P_{1}$ elements (QUODDY, ADCIRC, FESOM, ICOM) and $R T_{0}-P_{0}$ elements (triangular C-grid as used by UnTRIM and SUNTANS is just a special case) have been used most widely. In the $P_{1}-P_{1}$ case all DOFs are located at nodes, and fields are linearly interpolated on elements. In the second case $R T_{0}$ is the lowest-order Raviart-Thomas element (Raviart and Thomas (1977)). The normal velocity is specified at edges and the full velocities on triangles is the sum over edges

$$
\mathbf{u}_{t}=\sum_{e} u_{e} \phi_{e}, \quad \phi_{e}=\left(\mathbf{x}-\mathbf{x}_{e}\right) / h_{e}
$$

where $e$ lists edges of triangle $t, u_{e}$ is the normal velocity on the edge, $\mathbf{x}_{e}$ is the radius-vector drawn to the vertex opposing edge $e$, and $h_{e}$ is the distance from the vertex to the edge (the height of triangle). It is easy to see that the normal velocity is continuous across the edges, but tangent velocity is not. The elevation is $P_{0}$, i. e., elementwise constant.

Less frequent choice is $P_{1}^{n c}-P_{1}$ discretization (used in SLIM by White et al. (2008a) and also by Danilov et al. (2008)) in which case the velocity is represented with so-called non-conforming test functions $N_{e}^{n c}$ that equal one on edge $e$ and vary linearly to -1 on an opposing vertex (Hua and Thomasset (1984)). The velocity is only continuous at edge midpoints. Notice that $R T_{0}$ and $P_{1}^{n c}-P_{1}$ elements are already 'partly' discontinuous, and care should be 


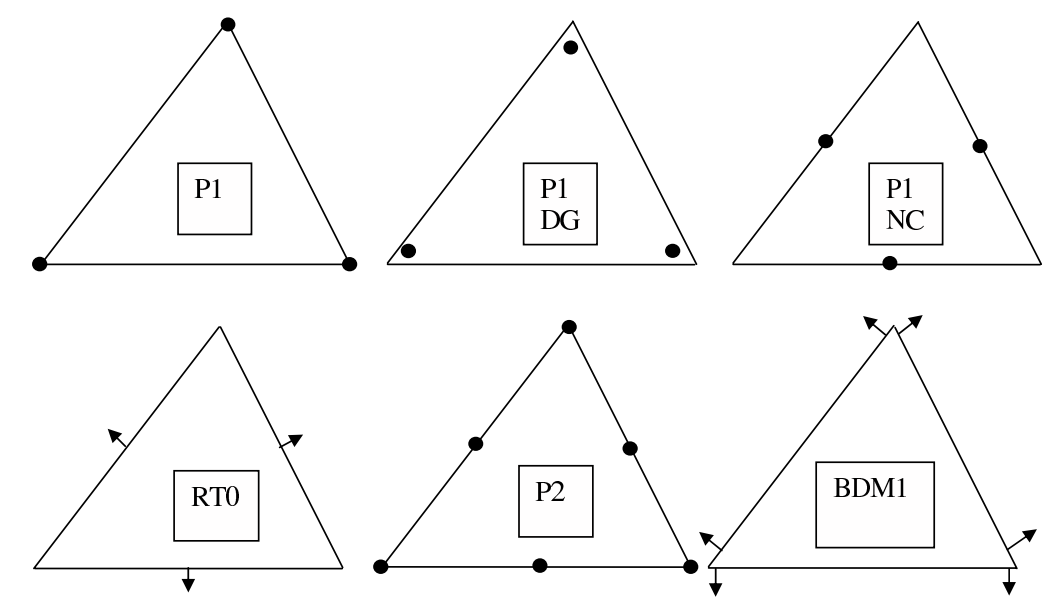

Figure 2: Placement of variables for several FE discretizations. Dark circles show the location of velocity or scalar variables, and the arrows show the location of normal velocities. The upper row, from left to right: $\left(P_{1}\right)$ Linear continuous representation, variables are at vertices; $\left(P_{1}^{D G}\right)$ Same location, but linear representation is restricted to elements and hence discontinuous across the edges, as a consequence each vertex hosts many DOF (6 in most cases); $\left(P_{1}^{n c}\right)$ Nonconforming linear representation, variables are at mid-edges, their basis functions change from 1 to -1 on an opposing vertex, continuity is maintained only at mid-edges. The lower row, from left to right: $\left(R T_{0}\right)$ Linear representation of velocity in terms of radial functions (6), the normal velocity is uniform on edges and continuous across them; $\left(P_{2}\right)$ Quadratic continuous representation, DOFs are at vertices and midedges; $B D M_{1}$ The velocity is linear on elements, normal velocity is linear and continuous at edges. $P_{0}$ (not shown here) is discontinuous and implies elementwise constant fields.

taken with respect to properly writing the discretized equations (see, e. g., Hanert et al. (2005) and Comblen et al. (2010)).

For discontinuous Galerkin $P_{1}^{D G}-P_{1}^{D G}$ discretization linear representation is confined to triangles (working applications are reported by Dawson et al. (2006), Blaise et al. (2010) and Kärnä et al. (2013)). Bernard et al. (2007) discuss higher-order possibilities. Since on good quality meshes in most cases 6 triangles meet at each vertex, $P_{1}^{D G}$ representation implies a 6-fold increase compared to CG $P_{1}$ representation in the number of DOFs in the horizontal direction. The factor is reduced if we compare polynomials of higher order, being 3 and 20/9 for the quadratic and cubic cases respectively. In essence, it characterizes clustering of DOFs in space which is rather high for the low-order DG discretizations on triangular meshes. As a result, they do not necessarily offer spatial resolution matching their higher com- 

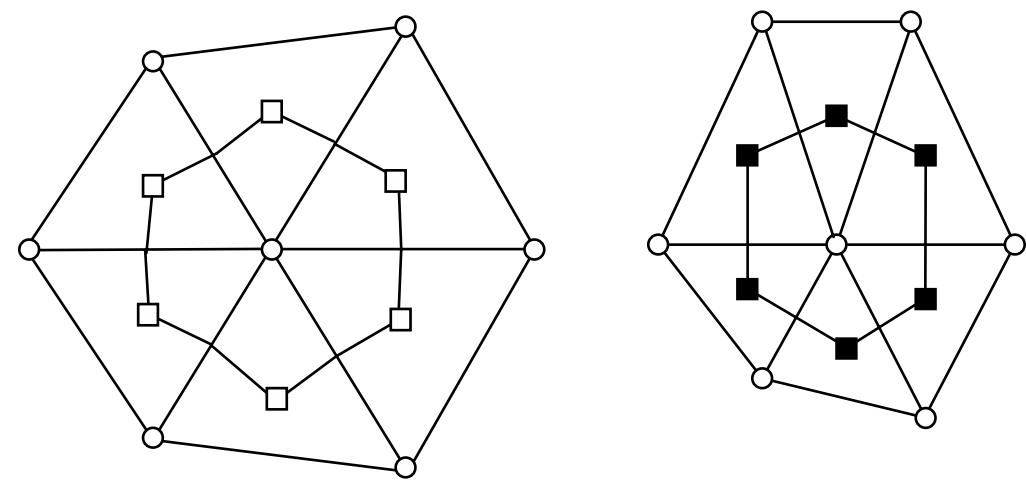

Figure 3: Placement of variables and control volumes for several FV discretizations. The circles, squares and dark squares mark, respectively, the vertices, centroids and circumcenters. The cell placement of variables implies centroids except for C-grids, when circumcenters are used. The control volumes are the elements proper. For vertex placement of variables, the control volumes are obtained by connecting either centroids with mid-edges (median-dual control volumes, left panel) or the circumcenters (right panel). The latter case corresponds to the Voronoi dual meshes. In that case the mesh is made of Voronoi cells (polygons with vertices at dark squares; they are hexagons in most cases). On triangular C-grids the normal velocities (not shown) are located at mid-edges. On Voronoi (quasi-hexagonal) meshes (right panel) they have the same location, but are normal to edges of hexagons, which are the lines connecting circumcenters of triangles.

putational cost with respect to their CG counterparts. This already hints that the DG FE method needs high-order elements to fully demonstrate its potential.

$P_{0}-P_{1}$ and $P_{1}^{D G}-P_{2}$ elements are two choices well suited to represent the geostrophic balance (because the pressure gradient and rotated pressure gradient lie in the velocity space). The lower-order one is used by FVCOM in the FV implementation. The higher-order one is currently used by ICOMFluidity. Its performance on the level of barotropic shallow water equations was explored by Cotter et al. (2009), Comblen et al. (2010) and Cotter and Ham (2011). Notice that it requires more than 3-fold increase in the number of DOF compared to the lower-order one.

There are many other possibilities yet they are without a practical record. Rostand and Le Roux (2008) considered generalizations of $R T_{0}-P_{0}$, one with $P_{1}$ elevation $\left(R T_{0}-P_{1}\right)$, and two others, where the velocity is represented by Brezzi-Douglas-Marini elements $\left(B D M_{1}\right.$, the normal velocity is linear and continuous at edges), and elevation as $P_{0}$ and $P_{1}$ respectively. 
Spurious elevation modes were identified for $P_{1}$ representations, and noise in the velocity field was observed for $B D M_{1}$ on unstructured meshes. There is no obvious recommendation with respect to these elements. Cotter and Shipton (2012) proposed to enrich $B D M_{1}$ and work with BDFM1- $P_{1}^{D G}$ pair, but no practical tests are known to us. Comblen et al. (2010) explore several discontinuous formulations such as $P_{1}^{n c}-P_{1}^{n c}$ and $P_{1}^{D G}-P_{1}$ in shallow-water tests. Of them $P_{1}^{n c}-P_{1}^{n c}$ looks promising because it needs twice less DOFs than $P_{1}^{D G}-P_{1}^{D G}$ but behaves rather similarly.

Le Roux et al. (2007) explored rather exotic variants such as $P_{1}^{n c}-P_{0}$ and $P_{2}-P_{0}$ but found them unsuitable for modeling surface inertia-gravity waves. The physical reason is that the stencil of $P_{1}^{n c}$ functions spans only two neighboring triangles sharing an edge, it is too small to compute gradients of $P_{0}$ elevation, hence noise. In the other case the velocity degrees of freedom associated with edges suffer from the same problem.

Since $P_{1}-P_{1}$ discretization may develop pressure modes, some modifications have been proposed with an enriched velocity space. One choice is the so-called MINI-element, where an additional basis function localized on element is introduced (frequently it is a cubic bubble that equals one at centroid and zero at the element boundary). Another possibility is $P_{1} i s o P_{2}-P_{1}$ pair (Le Roux et al. (1998)) where additional nodes are introduced at mid-edges, and each triangle is split in four for linear velocity representation (abbreviation $P_{1}$ iso $P_{2}$ reflects the fact that the number of DOF involved in this case is equal to that of quadratic $P_{2}$ representation). With, perhaps, the exception for TELEMAC (that uses quasi-bubble stabilization) we are unaware of other applications.

\subsubsection{Finite-volume discretization}

As mentioned above, there are two basic variable placements - cell centered and vertex-centered. In the first case the control volumes are the mesh elements (triangles, quads or hexagons). In the second case one commonly uses median-dual control volumes obtained by connecting centroids of elements with mid-edges (left panel of Fig. 2). Szmelter and Smolarkiewicz (2010) suggest to apply the second type of variable placement in geospherical context on triangular meshes. Because of its stencil it turns to be very close to $P_{1}-P_{1}$ FE discretization and shares the same difficulties (see further). MIKE 21 \& MIKE 3 Flow Model FM (http://www.mikebydhi.com) use cell-centered placement of all variables. FVCOM uses staggered representation, its velocities are at centroids, but scalar quantities are at vertices. 
This is very similar to the $P_{0}-P_{1} \mathrm{FE}$ case, with the difference that mass matrices are diagonal. We also note that the so-called ZM-grids on hexagonal surface meshes (Ringler and Randall (2002)) are very close to this discretization. The cell-vertex triangular discretization would be identical to ZM if the median-dual control volumes are replaced by the 'orthogonal' ones obtained by connecting circumcenters.

A special class of codes uses C-grid ideology on triangular meshes, keeping normal velocities at edges, and scalar fields at circumcenters (UnTRIM, SUNTANS and the model by Stuhne and Peltier (2006)). As concerns the scalar equations, the approach is FV. However, with respect to momentum equations, it applies finite-differences (computations of pressure gradient) and, in some codes, also FV (computations of momentum advection and viscosity). It presents a particular variant of mass matrix lumping of the $\mathrm{FE}$ $R T_{0}-P_{0}$ case. Same variable placement is used by the mimetic approach explored within ICON project (P. Korn 2011, personal communication). In that case one uses reconstruction (projection) operators from normal velocities on edges to full velocities on elements $(\mathrm{P})$ and back $\left(\mathrm{P}^{T}\right)$, and another set for the reconstruction to vertices (used for the Coriolis force). The simplest implementation of such operators coincides with that of Perot (2000). The key difference of mimetic approach from the pure C-grid is that $\mathrm{P}^{T} \mathrm{P} v_{e}$, where $v_{e}$ are normal velocities on edges, and not $v_{e}$, satisfy the continuity equation.

Unstructured-mesh C-grids are not limited to triangles and both quadrilateral and hexagonal C-grids offer clear advantages over triangles (see, Gassmann (2011) for comparison of triangular and hexagonal C-grids). For Voronoi meshes Thuburn et al. (2009) and Ringler et al. (2010) proposed the approach with mimetic properties, which will be referred further as TRiSK. Its essence lies in the reconstruction procedure for the tangential velocity component which allows to construct differential operators which mimic the behavior of their continuous analogs. This approach is pursued by MPAS initiative and shows a very robust performance. Gassmann (2012) offers some modifications to vorticity reconstruction that is motivated by atmospheric applications.

\subsection{Simple general view}

A question naturally arises as why so many approaches have been proposed. A very rough answer is that neither is perfect, and our aim here is to explain this situation on an elementary level. 
We begin with mentioning that there are two geometrical aspects associated with triangular and hexagonal meshes: (i) the disparity between the number of DOFs used to represent the horizontal velocity and scalar fields for most of staggered discretizations and (ii) the presence of DOFs with different neighborhood (like vertex and edge DOFs for $P_{2}$ elements) which may lead to 'grid imprinting' in eddy-dominated regimes aimed by large-scale applications. Here we mean the potential danger of noise from the build-in non-uniformity on the mesh scale in eddy-dominated regimes. This issue, however, remains unexplored.

For quadrilateral-grid models formulated on the Arakawa A, B or C grids the number of DOFs for the horizontal velocity is related to those of a scalar field as 2:1. Although the pressure modes are known to exist on A and B grids, and the Coriolis operator may have null-space on C-grids, these issues can be well handled on B and C-grids. Linearized shallow-water equations on an f-plane, discretized on A-, B- or C-grids, support one geostrophic and two inertia-gravity modes, as in the case of continuous equations. Additionally, the ratio of 2:1 implies that the spatial resolution of velocity and scalar fields is the same. Let us look from this perspective on the situation on triangular and hexagonal meshes. If the number of vertices $N$ on a triangular mesh is sufficiently large, the numbers of triangles and edges are approximately $2 N$ and $3 N$, respectively. On hexagonal meshes, if $N$ is the number of hexagons, $2 \mathrm{~N}$ is the approximate number of vertices and $3 N$ is that of edges. It is thus straightforward to see that the discretizations discussed above correspond to ratios given in Table 1. References there should help to find information, they do not reflect priority. The numbers correspond to degrees of freedom needed by discretizations on the level of shallow water equations.

From this table it follows that with exception of the recently proposed $B D F M_{1}-P_{1}^{D G}$, only the discretizations with same (collocated) representation for velocity and scalars $\left(P_{1}-P_{1}\right.$, its FV analog - vertex-based discretization of Szmelter and Smolarkiewicz (2010), cell-cell, $P_{1}^{D G}-P_{1}^{D G}$ and recently proposed $P_{1}^{n c}-P_{1}^{n c}$ ) realize this ratio. Note that except for $P_{1}-P_{1}$ and the cell-cell case (aiming at coastal applications) all other still need additional $\left(P_{1}^{D G}-P_{1}^{D G}\right)$ or fundamental efforts toward full ocean models. The rest of discretizations are 'unbalanced'. $R T_{0}-P_{0}$ and triangular C-grid possess too large scalar spaces, while all other discretizations have too many velocities. A large velocity space is as a rule needed to avoid the null space of gradient operator (pressure modes) which is the major drawback of $P_{1}-P_{1} \mathrm{FE}$ and vertex-based FV discretizations (as well as other collocated discretizations). 
Table 1: Ratio of degrees of freedom (horizontal velocity : scalar field)

\begin{tabular}{|l|l|l|}
\hline Discretization & Ratio & Reference \\
\hline$P_{1}-P_{1}$ & $2 N: N$ & ADCIRC,FESOM,ICOM \\
\hline vertex-vertex & $2 N: N$ & Szmelter and Smolarkiewicz (2010) \\
\hline cell-cell & $4 N: 2 N$ & MIKE 21 \\
\hline$P_{1}^{D G}-P_{1}^{D G}$ & $12 N: 6 N$ & Blaise et al. (2010) \\
\hline$P_{1}^{n c}-P_{1}^{n c}$ & $6 N: 3 N$ & Comblen et al. (2010) \\
\hline$B D F M_{1}-P_{1}^{D G}$ & $12 N: 6 N$ & Cotter and Shipton (2012) \\
\hline Tri-C-grid & $3 N: 2 N$ & Casulli and Walters (2000) \\
\hline$R T_{0}-P_{0}$ & $3 N: 2 N$ & Walters et al. (2009) \\
\hline Hex-C-grid & $3 N: N$ & Ringler et al. (2010) \\
\hline$P_{1}^{D G}-P_{2}$ & $12 N: 4 N$ & Cotter and Ham (2011) \\
\hline$R T_{0}-P_{1}$ & $3 N: N$ & Rostand and Le Roux (2008) \\
\hline$B D M_{1}-P_{0}$ & $6 N: 2 N$ & Rostand and Le Roux (2008) \\
\hline cell-vertex & $4 N: N$ & FVCOM \\
\hline$P_{0}-P_{1}$ & $4 N: N$ & Le Roux et al. (2007) \\
\hline Hex-ZM-grid & $4 N: N$ & Ringler and Randall (2002) \\
\hline$P_{1}^{n c}-P_{1}$ & $6 N: N$ & Hanert et al. (2005) \\
\hline MINI- $P_{1}$ & $6 N: N$ & Le Roux et al. (2007) \\
\hline$B D M_{1}-P_{1}$ & $6 N: N$ & Rostand and Le Roux (2008) \\
\hline$P_{2}-P_{1}$ & $8 N: N$ & Le Roux et al. (2007) \\
\hline$P_{1}^{D G}-P_{1}$ & $12 N: N$ & Comblen et al. (2010) \\
\hline
\end{tabular}

One expects spurious numerical modes for 'unbalanced' discretizations, and it is indeed so. A dominant part of the discussion of element pairs in the literature relies on linearized barotropic shallow water equations. Assuming regular triangulation and plane geometry, one examines the behavior of a Fourier mode, similarly to the analyses on reqular quadrilateral meshes. Additional insight is provided by selecting unstructured meshes of limited size and performing analyses of dicrete operators. Le Roux (2005), Le Roux et al. (2007), Le Roux and Pouliot (2008), Bernard et al. (2008), Bernard et al. (2009), Hanert et al. (2009), Walters et al. (2009) and Cotter and Ham (2011) (see also references therein) explore different aspects of gravity and Rossby wave propagation for various discretization types, and Thuburn (2008) gives the analysis for hexagonal meshes. Recent study by Le Roux (2012) provides an excellent summary of the effect of spurious iner- 
tial modes. The details are too numerous to be given here and would require a review on their own. Briefly, except for pathological discretizations like $P_{1}^{n c}-P_{0}$, the rest are capable of representing waves with desirable properties (accuracy and sensitivity to mesh structure vary between discretizations). However, many of them support spurious numerical modes. Different from the pressure modes on the Arakawa A and B-grids, emerging for isolated wave numbers, here we encounter spurious numerical branches. The most important question is about their consequences.

There are additional subtleties related to the ability of discretizations to maintain the geostrophic balance as explained by Le Roux et al. (1998) and Bernard et al. (2008). For example, it turns out that unstabilized $P_{1}-P_{1}$ representation is suboptimal for that on deformed meshes (yet it is never used without stabilization). Some details will be presented further.

Unfortunately, the presence of spurious branches for 'unbalanced' discretizations may have implications beyond the shallow water equations, so that full 3D setups are required to learn about them. 'Balanced' collocated discretizations are analogous to A-grids and need special measures to suppress pressure modes. Finally, the 'balanced' mixed discretizations like $B D F M_{1}-P_{1}^{D G}$, may suffer from 'grid imprinting' in strongly nonlinear regimes, as it introduces two types of velocity degrees of freedom. This also concerns some 'unbalanced' discretizations listed above. There is a parallel discussion of certain issues mentioned here in the atmospheric community, and a review by Staniforth and Thuburn (2011) provides many relevant details.

This highlights the difficulties, and we just add some details.

\subsubsection{Spurious modes}

Table 3.1 in Le Roux et al. (2007) and Table 3 in Le Roux (2012) list numerical (physical and spurious) modes for many discretizations, the latter reference also presents general rule to compute the number of spurious inertial modes. Here we only give some illustrations.

$R T_{0}-P_{0}$ and triangular C-grid support four coupled inertia-gravity modes (see Le Roux et al. (2007), Gassmann (2011)), two of which can be identified with physical modes if the Rossby radius is well resolved. Otherwise the separation into physical and spurious parts fails. In typical barotropic simulations the external Rossby radius is well resolved, and spurious modes are not excited. But situation is different when dynamics are baroclinic. The horizontal divergence that corresponds to eigenvectors of spurious modes (or 
any of four modes if resolution is coarse) shows a checkerboard pattern, which projects on the field of vertical velocity. Accordingly, these discretizations become a questionable choice for large-scale ocean modeling (see Danilov (2010)), despite their obvious algorithmic simplicity and despite the fact that they are widely used for coastal simulations. To suppress numerical modes, some form of divergence averaging is needed. Averaging of velocity and elevation gradient by the operator $\mathrm{P}^{T} \mathrm{P}$ in ICON-ocean may serve this purpose. These measures effectively reduce the resolution and modify the sense in which the local volume conservation has to be understood.

The discretizations with large velocity space support in many cases only spurious inertial velocity modes (as is the case with $P_{1}^{n c}-P_{1}, P_{1}^{D G}-P_{2}$, $P_{0}-P_{1}$, or cell-vertex scheme of FVCOM - i. e., the discretizations with full horizontal velocity vectors). On their own these modes are not dangerous in linear problems if damped by dissipation in the momentum equations (yet may become dangerous if excited by nonlinear dynamics). Le Roux (2012) shows that they are in many cases responsible for the reduced convergence in solutions without dissipation.

The hexagonal C-grid has two coupled geostrophic modes which are sensitive to the implementation of Coriolis operator. Only if special care is exercised, the geostrophic modes become stationary on an $f$-plane, but there still remain two coupled branches of Rossby waves if the Coriolis parameter varies. Luckily, one of them is close to the physical mode at small wavenumbers (see Thuburn (2008), Thuburn et al. (2009)). Similarly, the generalizations introduced by Rostand and Le Roux (2008) all have coupled geostrophic modes, which should have implications for Rossby waves. The general feature of discretizations introducing only normal components of velocity is the absence of inertial modes.

\subsubsection{Momentum advection}

The too large velocity space size of certain discretizations has furtherreaching implications in eddying regimes, when momentum advection is no longer small. Indeed, the mere fact that the velocity space is too large implies that it resolves scales smaller than those of pressure gradient. In turn, due to nonlinearity, even smaller scales are produced. They have to be effectively removed to maintain numerical stability, which in practice requires designing special algorithms (see, for example, Danilov et al. (2008) for $P_{1}^{n c}-P_{1}$ case and Danilov (2012) for cell-vertex discretization; see also discussion of ZM grid by Ringler and Randall (2002)). Standard Laplacian viscosity is fre- 
quently insufficient (cell-vertex) or should be unrealistically high $\left(P_{1}^{n c}-P_{1}\right)$. One runs into a paradoxical situation: the extra velocity DOFs, needed to prohibit pressure modes, must in the end be filtered out; there is no real benefit from keeping them. Le Roux (2012) recommends using discretizations with collocated scalars and horizontal velocities suggesting that it is easier to stabilize against pressure modes than to remove the consequences of inertial modes.

We note that the measures to stabilize the momentum advection may depend on the form it is written. For the flux form, upwinding and flux limiting can be used to dissipate grid-scale velocity. For the vector invariant form, filtering can be done for the relative vorticity and kinetic energy. It should also be taken into account that the relative vorticity and kinetic energy are defined at different locations than the velocity. This alone may lead to filtering, as is the case for the cell-vertex discretization, see section 7.3.

\subsubsection{Pressure modes and summarizing remarks}

The frequently used 'balanced' $P_{1}-P_{1}$ (or vertex-vertex) discretization has no obvious problem with the momentum advection but is notoriously famous for its pressure modes linked to the non-trivial null space of the discrete gradient operator. Although the null space can be removed if the boundary condition of impermeability is imposed weakly (Hanert and Legat (2006)) or can be absent on irregular meshes, in practice such codes still require some form of stabilization (there are several variants, and ADCIRC, FESOM and ICOM implementation by Piggott et al. (2008) exemplify different possibilities; see Le Roux et al. (2012) for the analysis of consequences of one particular method). The origin of difficulty is easy to grasp - even if the true null-space is absent, the operator occurring in the discrete wave equation ( $G^{T} H M^{-1} G$, where $H$ denotes vertical integration, $M$ the mass matrix and $G$ the gradient) still has small eigenvalues. (For diagonally approximated mass matrices it turns out to be defined on a stencil involving neighbors of neighbors, so it does not penalize features on the mesh scale.) The system fails if such scales are triggered, for example, through inhomogeneous topography, especially on $z$-coordinate meshes. Notice that DG FE $P_{1}$ discretization and recently suggested (discontinuous) $P_{1}^{n c}-P_{1}^{n c}$ Comblen et al. (2010) handle these difficulties by using upwinding of fluxes. Although stabilizations can be tuned to be at minimum compatible with the code stability, they always have implications for energetic consistency and, in certain variants, also for volume and tracer balances. 
Summing up, it is rather difficult to suggest an equivocally winning discretization among those having practical records. Judged by supported modes and bearing in mind tasks of large-scale ocean modeling, preference should be given to pairs without pressure or divergence modes, i. e. C-grid on hexagonal meshes or $P_{1}^{N C}-P_{1}$ or cell-vertex $\mathrm{FV}$ on triangular meshes. Neither of them is, however, balanced, and the last two require special measures to suppress the manifestations of too large velocity space. $P_{1}^{D G}-P_{1}^{D G}$ is balanced but needs to gain in numerical efficiency and prove its skill in large-scale setups. This, arguably, explains why unstructured-mesh modeling community in its significant part cannot converge to just a couple of discretizations (such as B or C-grids on regular quadrilaterals) and continues to search for more sophisticated variants (such as $P_{1}^{D G}-P_{2}$ in Cotter and Ham (2011), $P_{1}^{n c}-P_{1}^{n c}$ in Comblen et al. (2010) and recently proposed $B D F M_{1}-P_{1}^{D G}$ in Cotter and Shipton (2012); the last two, however, wait for practical records).

The real situation proves to be even more complicated. In FE hydrostatic models the representation of elevation dictates the representation for other scalars, as discussed in the next section. This introduces some unwanted features on continuous elements, making them a suboptimal option for future development.

\section{Conservation and consistency properties}

\subsection{Notes on conservation}

Conservation properties of CG FE codes are based on the variational formulation, and of FV and DG FE codes, on their flux form. This implies that obvious balances (volume, tracer, momentum and, to a certain extent, energy) are guaranteed by construction. More delicate balances involving enstrophy are not always possible on the discrete level in CG FE codes working with the primitive equations (because the projection on test functions and curl operator not necessarily commute). Some FV discretizations can maintain discrete vorticity balance if the momentum equations are written in the vector-invariant form (e. g., C-grid, see Thuburn et al. (2009) and Ringler et al. (2010), or cell-vertex, which can be proved in analogy to Ringler and Randall (2002)) and indeed respect mimetic properties, and some not (curl of pressure gradient $\nabla p$ is not necessarily zero for vertex-vertex approach of Szmelter and Smolarkiewicz (2010)). Additional issues are linked 
with maintaining symmetry between discrete gradient and divergence operators (so that one is the minus transpose of the other in the energy norm), which is automatically achieved in CG FE codes, but requires care in the FV and DG FE cases. Note that this symmetry is broken in codes introducing stabilization against pressure modes. Note also that in most coastal codes the momentum advection is taken in the flux form (see, e. g., Chen et al. (2003) or Fringer et al. (2006)), and this approach is also followed by FESOM Wang et al. (2008); MPAS-ocean uses the vector-invariant form of momentum equations and enstrophy conserving implementation, while the cell-vertex code in Danilov (2012) can use both forms. Merits of different momentum equation forms are discussed in Ringler (2011).

It should be reminded that the local volume and tracer conservation in CG FE codes is expressed in a cluster-weighted form instead of flux form one is inclined to have. This leads to uncertainties in interpreting transports computed directly, as discussed by Sidorenko et al. (2009). Although uncertainties disappear as resolution is improved, they are frequently annoying in practice if weak transport variability is studied.

\subsection{Space consistency requirements in FE codes}

In hydrostatic FE codes the space selected for the elevation defines the horizontal representation of vertical velocity, tracers and pressure. In particular, $P_{1}$ or $P_{2}$ continuous elevation means same continuous horizontal representation for the vertical velocity, temperature, salinity and pressure fields. This has certain implications for CG FE discretizations, as partly mentioned in the foregoing analysis.

First, because of horizontal connections introduced by continuous basis and test functions $N_{i}$, the computation of vertical velocity or hydrostatic pressure involves global matrices. Moreover, the iterative solution for pressure leaves in some cases a mode which makes the horizontal pressure derivatives too noisy (leaving aside the fact that the overall performance is slowed down). One way out on prismatic meshes lies in applying horizontal lumping in the operator parts of equations on vertical velocity $w$ and pressure $p$, which removes the horizontal coupling (this requires some modifications in tracer and elevation equations for consistency, but leaves errors in the energy transfer). Additionally, if continuous linear functions are used in the vertical direction, odd and even vertical levels are coupled only at boundaries.

Existing codes tackle these issues in a set of approximations. The horizontal lumping is applied in FESOM on prismatic meshes (Wang et al. (2008)) 
and in SLIM version by White et al. (2008a). FESOM uses the ansatz $w=\partial_{z} \phi$ for the vertical velocity where $\phi$ is the vertical velocity potential, to override the odd-even decoupling and White et al. (2008a) resort to vertically discontinuous representation. ADCIRC also uses lumping, and finitedifferences for the vertical part of the operator. On tetrahedral vertically aligned meshes, the operator part of $\partial_{z z} \phi=-\nabla \mathbf{u}$ connects only vertically aligned nodes if $\phi$ is linear. Yet the $w$ found in this way has a tendency to noise unless the meshes are sufficiently smooth.

As concerns the pressure, spline interpolation is needed to minimize pressure gradient errors on generalized meshes unless high-order polynomials are used in the vertical direction. This destroys the energetic (space) consistency of $\mathrm{FE}$ codes and introduces imbalances in the energy conversion. Wang et al. (2008) present details of FESOM algorithm which is largely finite-difference in the vertical direction. Ford et al. (2004) split pressure into two contributions belonging to different spaces, so that the energetic consistency is also broken).

Second, as we have already mentioned, global mass-matrices appear in CG codes. Although they yield to fairly inexpensive iterative solution procedures and substantially improve the performance of advection, they still slow down the performance. The implicit vertical diffusion leads to global matrices too. Horizontal lumping decouples horizontal directions from vertical, but destroys true conservation. On vertically aligned tetrahedral meshes, $\partial_{z z}$ couples only vertically aligned nodes for $P_{1}$ continuous fields, but horizontal connections introduced by mass matrix still have to be resolved.

We thus see that using continuous FE to represent scalar quantities is not free of complications: the horizontal connections of CG FE are at variance with the structure of hydrostatic codes. Note that issues discussed here are independent on how well the wave propagation is simulated by a particular pair on the level of shallow water equations. The existing CG FE ocean circulation models are always resorting to some compromise solutions. While practical, they destroy the mathematical beauty of the FE method, and in reality the rigorous variational formulation is lost. This statement does not rule out the CG methods, it only points that they are difficult to implement in a rigorous way.

\subsection{Hydrostatic vs. nonhydrostatic}

Because of predominantly vertical stratification of the ocean and smallness of nonhydrostatic effects the current practice in ocean modeling treats 
the nonhydrostatic part as a correction to the hydrostatic one, as proposed by MITgcm Marshall et al. (1997) and followed by SUNTANS, FESOM, and recently by FVCOM (Lai et al. (2010)). This requires that the hydrostatic pressure and elevation lie in the same space as nonhydrostatic pressure correction.

In nonhydrostatic $\mathrm{FE}$ codes the vertical velocity $w$ belongs to the same space as the components of horizontal velocity, and the space for pressure can be selected independently. The logic of nonhydrostatic correction is then only compatible with equal interpolation for all variables.

ICOM (Ford et al. (2004), Piggott et al. (2008)) does not follow the concept of nonhydrostatic correction, but still splits pressure into two contributions residing in different spaces to ease the solution.

\subsection{Geostrophic balance}

As related to large-scale flows, there is a natural question whether the discretizations discussed here are capable of maintaining the geostrophic balance. The elementary aspect of this balance - the presence of stationary geostrophic mode in the dispersion relation of linearized f-plane shallowwater equations on regular triangular, quadrilateral and hexagonal meshes can be easily explored. Le Roux et al. (2007) and Le Roux (2012) consider many triangular discretizations discussed above and show that it is the case for most of them; an example of a pair that does not have a stationary geostrophic mode is $P_{2}-P_{0}$. A more difficult question is what happens when the mesh is irregular. TRiSK approach ensures the maintenance of stationary geostrophic mode by demanding that the discrete vorticity balance is observed and additionally that vorticity dynamics are stationary if divergence equals zero (f-plane). For some FE discretizations the geostrophic balance can be proven for arbitrary meshes based on geometrical considerations. They include $P_{0}-P_{1}, P_{1}^{D G}-P_{2}$ and the family of 'finite element exterior calculus', exemplified by $B D M_{1}-P_{0}$ and $B D F M_{1}-P_{1}^{D G}$, see Cotter and Ham (2011) and Cotter and Shipton (2012).

In a general case the kernel analysis and search for the smallest representable vortices (SRVs) proves to be helpful (see Rostand and Le Roux (2008) and Le Roux (2012)). Given the linearized shallow water equations on f-plane,

$$
\partial \mathbf{U}+f \mathbf{k} \times \mathbf{U}+c^{2} \nabla \eta=0, \quad \partial \eta+\nabla \cdot \mathbf{U}=0,
$$

one seeks for stationary solutions that simultaneously satisfy geostrophy and continuity. In a discrete form, such solutions have to satisfy $C \mathbf{U}^{h}+G \eta^{h}=$ 
$0, G^{T} \mathbf{U}^{h}=0$, where the distance is made dimensionless with the Rossby radius of deformation, $C$ and $G$ are, respectively, the Coriolis and gradient operators, and superscript $h$ denotes the discrete representation. A SRV is a solution with minimum support. Clearly, such solutions lie in the null space of $G^{T} C^{-1} G$ and thus can be considered as forming a basis for geostrophic flows. There should be sufficient number of them to ensure that the geostrophic balance is well represented. Since the velocity mass matrices are diagonal for $P_{1}^{N C}-P_{1}$ and $P_{0}-P_{1}$, their SRVs can easily be found geometrically by simply taking elevation be one at vertex $i$ and zero otherwise. The resulting flow $C^{-1} G \eta^{h}$ has zero divergence. The task is more delicate for $R T_{0}$ element as the Coriolis operator is not necessarily invertible in this case. However, a full set of SRV is found for it too. A problem occurs for those FE pairs that have continuous velocities and non-diagonal mass matrices, like $P_{1}-P_{1}$. In this case SRVs exist on regular meshes, but cease to exist on irregular meshes. Relatedly, discrete geostrophic solutions suffer from non-zero residual divergence. This correlates with errors in the Rossby wave dispersion demonstrated for such discretizations by Rostand and Le Roux (2008) and also with the absence of the discrete analogs of continuous identity $\nabla \times \nabla \eta=0$. As concerns $P_{1}-P_{1}$ discretization, it is seldom used without stabilization, which, couples inertia-gravity and geostrophic modes even on uniform triangular meshes.

Once again, the drawbacks do not necessarily rule out these discretizations as viscosity, nonlinearity and nonstationarity always maintain some deviations from geostrophy. They, however, signal about potential problems for their use in large-scale ocean modeling.

\section{Advection schemes}

The availability and computational cost of advection schemes with desirable properties offers one more criterion to judge about unstructured-mesh discretizations. Bearing in mind large-scale modeling tasks, one typically needs to maintain eddy dynamics on the fine part of computational mesh and preserve water-mass properties over large time intervals. Both demand advection schemes with low numerical dissipation and dispersion, which is often a synonym for higher accuracy. The question is what is possible to achieve with low-order discretizations.

There is vast literature on advection schemes designed for unstructured meshes, yet they are frequently method-specific (a FV scheme, e. g., is as a rule inapplicable for $\mathrm{FE}$ discretization) and not necessarily generalizable 
to three dimensions. A review by Budgell et al. (2007) analyzes the performance of some of them (belonging into FE, FV and DG FE classes) in two dimensions for low-order representations. It should be reminded that in the FE case the order of convergence is defined by the order of polynomial representation (it can be reduced if measures to maintain monotonicity are introduced), which is illustrated in Budgell et al. (2007). Importantly, the FE flux-corrected transport (FCT) scheme by Löhner et al. (1987) (CG $P_{1}$ ) was found to keep the second order while providing monotonicity of solutions. A review by Cueto-Felgueroso and Colominas (2008) discusses FV schemes on unstructured meshes with order higher than two, which, in the absence of mass matrices, are as a rule necessary in practice in this case. There is no limit on the method order, and the argument is rather the computational cost of further error reduction.

We briefly discuss several approaches related to CG FE and FV further.

\subsection{Streamline-upwind Petrov-Galerkin method}

Advection schemes of CG FE method are largely equivalent to central differencing. Consistent mass matrices reduce their dispersion and they show smaller phase errors than their FV counterparts. For practical usage they have to be augmented either with explicit isopycnal diffusion, FCT, or be stabilized in the spirit of streamline-upwind Petrov-Galerkin method (SUPG). The latter is equivalent to high-order upwinding. In the simplest case the test function is selected as $M_{i}=N_{i}+R N_{i}=N_{i}+\epsilon\left(\mathbf{u} \nabla N_{i}+w \partial_{z} N_{i}\right)$ where $\epsilon$ is the stabilization parameter with dimension of time. It is elementwise constant and is taken so that stabilization is on when advection dominates over explicit diffusion. The algorithm to select $\epsilon$ is a key ingredient, its optimal choice is not necessarily straightforward (some variants are cited in Budgell et al. (2007)). Our experience with FESOM which supports such a scheme is not in its favor. Partly the difficulty comes from disparity between $\mathbf{u}$ and $w$. The other part is the computational cost because the method leads to full 3D matrix problem. This method is frequently used in engineering. Its potential as applied to oceanographic tasks remains largely unexplored.

\subsection{FCT}

The FE FCT algorithm by Löhner et al. (1987) uses Taylor-Galerkin (Lax-Wendroff) approach with consistent mass matrices for the high order solution and adds artificial dissipation to obtain a low-order scheme. To a degree, the success of FESOM is based on this scheme which is explicit in 
time and robust in performance. Generalization of FCT algorithm toward minimum possible dissipation is proposed by Kuzmin and Turek (2002).

Practical difficulty of FE FCT as applied to continuous FE is that all nodes of numerical stencil contribute simultaneously to horizontal and vertical fluxes (flux here is the contribution of advection on a given element to its nodes). The limiting procedure is then based on maximum and minimum of low-order solution over the entire stencil, which mixes horizontal and vertical directions. Since vertical stratification is frequently much stronger, one cannot ensure that horizontal over- and undershoots are removed. As a result, true monotonicity is not reached. Further work in this direction is required.

On tetrahedral meshes, 3D numerical stencils may vary substantially from node to node which leaves certain grid-scale noise in the low-order solutions obtained by the algorithm of Löhner et al. (1987). The algorithm by Kuzmin and Turek (2002) performs slightly better in this respect.

FV implementations of FCT are not different from those on structured meshes. For geometrical reasons, there are more flux contributions to a scalar cell on vertex-based and hexagonal meshes than on quadrilateral meshes, and both horizontal directions have to be treated together. This explains why the FCT on unstructured meshes is more expensive than on regular meshes.

\subsection{High-order FV schemes}

The accuracy of FV advection schemes depends on how accurately the divergence of fluxes through the faces of control volumes is estimated. A widely used technology resorts to accurate field reconstructions. Consider reconstruction in the horizontal plane on control volume $i$ of triangular mesh (vertical direction is not specific). One seeks the representation $\mathcal{T}_{i}(\mathbf{r})=$ $\overline{T_{i}}+(\nabla T)_{i} \cdot\left(\mathbf{r}-\mathbf{r}_{i}\right)+\ldots$ on the cell around node $i$ (for vertex-based scalars) or in element $i$ (for cell-based scalars) imposing the strong constraint $\int_{i} \mathcal{T}_{i} d \mathbf{r}=$ $S_{i} T_{i}$ and minimizing the deviations over neighboring control volumes. This requires solution of the constrained least squares problem. Here $\mathbf{r}$ and $\mathbf{r}_{i}$ are radius vectors drawn, respectively, to an arbitrary point and either the vertex $i$ or centroid of cell $i$, and $S_{i}$ is the control volume area. One needs to find in general case 3 unknowns for a linear reconstruction, six for the quadratic one and ten for the cubic. For vertex control volumes the nearest neighborhood as a rule includes six control volumes, which is sufficient for the second order reconstruction. For the cell control volumes, there are only three nearest neighbors (which share edges), and the next level is frequently sufficient for a cubic reconstruction. Ollivier-Gooh and Van Altena (2002) and Ouvrard 
et al. (2009) provide the general description of the method, and Skamarock and Menchaca (2010) report on test results on hexagonal meshes (similar to vertex triangular), with the conclusion that quadratic reconstruction is optimal judged by accuracy against the computational effort. Quadratic reconstruction formally leads the third order scheme.

Simplest in this hierarchy are the scheme by Miura (2007) and the upwind scheme of FVCOM, which are based on a linear reconstruction. For the vertex variable placement they are noticeably less accurate than the $P_{1} \mathrm{FE}$ FCT scheme by Löhner et al. (1987). Indeed, since reconstructions operate with a gradient on the entire control volume, they smooth actual gradients on triangles removing the scales of the mesh size, and the rest is due to consistent mass matrices in the FE case. This points to the need of higher-order reconstructions in FV codes, in accordance with Skamarock and Menchaca (2010). The scheme by Miura (2007) belongs to the so-called direct spacetime schemes which estimate fluxes by approximately computing the amount of tracer in a volume of fluid that crosses the face during the time step. The scheme proposed by Lipscomb and Ringler (2005) is similar in spirit but relies on incremental remapping. While more computationally demanding, it may incorporate limiting in the reconstruction phase, thus avoiding the need and expense of FCT. Moreover, it will even become more economical in applications working with many tracers as the geometric information needed for remapping is computed only once per time step.

Another approach, described by Abalakin et al. (2002), exploits the idea of gradient reconstruction in a manner that provides high accuracy of not the flux, but flux divergence. The reconstruction mixes centered and upwind estimates and is in fact used by many finite-difference schemes (like Hundsdorfer and Spee (1995) or improved schemes by Webb et al. (1998)). The approach ensures that the scheme is second-order but becomes thirdor higher-order on uniform meshes. Skamarock and Gassmann (2011) suggest a very similar idea for hexagonal meshes, yet expressed differently, and test it showing that it competes favorably with schemes based on high-order reconstructions. Systematic studies of schemes mentioned in this section on non-uniform meshes are absent.

\section{More on practical examples}

The discussion above explains why the development of unstructured-mesh ocean circulation followed many roads. Indeed, the significance of many 
issues was appreciated through experimenting with the existing setups. Here we return to the main available approaches, trying to minimize the repetition of previous material.

As is clear from the discussion above, unstructured meshes maintain analogs of Arakawa A (all arrangements with same placement of velocity and scalars) and $\mathrm{C}$ grid discretizations. There is no true analog to B-grid or C-D-grid, but the cell-vertex, ZM or $P_{1}^{n c}-P_{1}$ discretizations resemble them to some extent through staggering and keeping full horizontal velocities. We will follow this template.

\subsection{A-grids}

FESOM, ADCIRC, QUODDY and previous versions of ICOM all have an A-grid placement of variables in the horizontal plane. They all need stabilization against pressure modes. Even in situation when the gradient operator has a full rank, pressure modes are easily triggered by bottom topography, especially on z-meshes. The methods used to suppress pressure modes have much in common with that discussed by Killworth et al. (1991) for the Arakawa B-grid. They modify the treatment of vertically integrated (or full) continuity equation, which may have implications for the volume conservation. The popular stabilization technique exploits the generalized wave continuity equation instead of the true continuity. This is a frequent option in coastal and tidal applications (e. g., ADCIRC). It introduces inconsistency between 2D and 3D interpretations of continuity (for discussion, see Massey and Blain (2006)). The stabilization used in FESOM (Wang et al. (2008)) maintains volume and tracer conservation but on the expense of some uncertainty in the momentum equations. ICOM/Fluidity uses nonhydrostatic solver and modifies full continuity equation when working with $P_{1}$ elements (Piggott et al. (2008)).

On the mathematical side, the need for stabilization is discouraging. In addition to the volume and tracer conservation issues, stabilization is incompatible with exact energy balance on the discrete level. The imbalance in the energy transfer between the available potential and kinetic energies is not negligible in certain cases (see, e. g., Danilov (2012)). In practice, however, the drawbacks of stabilization are not immediately apparent. ADCIRC enjoys obvious recognition as a tool for coastal applications. On large-scales, FESOM shows robust performance and simulates under COREI forcing (Sidorenko et al. (2011)) an ocean state similar to that of other model participating in COREs (Griffies et al. (2009)). 
An FV implementation of $P_{1}-P_{1}$ approach (vertex-vertex control volumes) was tried by Danilov (2012), triggered by the work by Szmelter and Smolarkiewicz (2010). It turns out to be more economical in terms of CPU time, suggests more freedom with respect to advection schemes, yet needs the same type of stabilization as FESOM on $z$-coordinate bottom. The cell-cell setup of MIKE $21 \&$ MIKE 3 is an alternative implementation of A-grid. We do not have sufficient information to discuss it.

Because of nodal placement of $P_{1}$ velocities, the no-slip boundary condition is the only safe option on $z$-meshes. This adds friction in narrow straits, and in fact implies that straits need to be better resolved than on C-grids.

Imperfections of triangular A-grids prompted work on setups free of pressure modes. Different ways are followed. The ADCIRC community explores the potential of discontinuous methods (Dawson et al. (2006)), and the same road is taken by SLIM (Blaise et al. (2010), Kärnä et al. (2013)). In the framework of FESOM, $P_{1}^{n c}-P_{1}$ discretization was tried (Danilov et al. (2008)), together with the cell-vertex FV setups. They will be mentioned further.

\section{2. $C$-grids}

UnTRIM, ELCIRC, SUNTANS, and the model by Stuhne and Peltier (2006) follow the triangular C-grid ideology. Models that exploit $R T_{0}-P_{0}$ element are rather similar to them but more general. They introduce a mass matrix for velocity. Walters et al. (2009) discuss two versions of mass matrix lumping, one of which reduces the $R T_{0}-P_{0}$ discretization to the triangular C-grid. The other one looks similarly, but replaces the distance between circumcenters by the distance between centroids along the edge normal. References to earlier implementations can also be found there. Numerous coastal applications performed with models based on triangular C-grids witness in favor of this approach (they are not cited here). However, on long time scales, as already mentioned, triangular C-grids generate strong noise in the field of horizontal divergence and hence vertical velocity. The noise is rooted in the too large size of the discrete horizontal divergence space, which leads to coupling between spurious and physical modes of inertia-gravity waves mentioned earlier (Gassmann (2011), Danilov (2010)). This makes triangular $\mathrm{C}$-grid or $R T_{0}$ models hardly suitable to large-scale ocean modeling unless measures leading to divergence smoothing are applied. Such measures are discussed by Wan et al. (2013) in the context of ICON-atmosphere (strong biharmonic viscosity with specially selected amplitude), Wolfram and Fringer 
(2013) (implicit velocity filters) and they are also pursued by the mimetic approach by P. Korn (private communication) utilized by ICON-ocean.

Triangular C-grids work only on orthogonal meshes (circumcenters are inside triangles). The $R T_{0}-P_{0}$ approach is formally free of this constraint but on the expense of mass matrices. The second lumping scheme works for general meshes too, but is less accurate (Walters et al. (2009)).

On hexagonal C-grids the number of scalar degrees of freedom is twice smaller, and the divergence noise is not generated. For that reason they present a potentially much better alternative than triangles as concerns largescale flows, and are in fact one of most promising discretizations for largescale modeling. It should be reminded that in the case of variable resolution we are dealing with Voronoi meshes that may include some amount of other polygons in addition to hexagons. The antisymmetry of Coriolis operator and stationarity of geostrophic mode on arbitrary Voronoi meshes require care, but they are well handled by the TRiSK reconstruction scheme (Thuburn et al. (2009), Ringler et al. (2010)). This scheme is only zeroth-order accurate on variable resolution meshes which demands that the mesh resolution varies smoothly. Errors can be amplified locally, for example, when different types of polygons meet together. A quasi-hexagonal C-grid unstructured-mesh ocean is current focus of MPAS project, and the already available results (Ringler et al. (2013) show that it has all necessary skills).

\subsection{Quasi-B-grids}

As mentioned above, there are no true B-grid analogs on triangular meshes, and the name of quasi-B-grid will be applied to the approaches that introduce full horizontal velocity vectors and staggering. On the FE side, an example is furnished by $P_{1}^{n c}-P_{1}$ elements, and on the FV side, by the cell-vertex FV discretization.

The attention to $P_{1}^{n c}-P_{1}$ discretization was drawn by a barotropic shallow water model by Hanert et al. (2005). Later this discretization served as the basis of 3D shallow-water model in the framework of SLIM (White et al. (2008a)) and was also explored by Danilov et al. (2008) as an alternative for FESOM $P_{1}-P_{1}$ discretization.

The study by Hanert et al. (2009) explores further the convergence properties ensured by this discretization in the shallow water context to note that it drops from the second to first order for the elevation on unstructured meshes. Bernard et al. (2009) similarly point to the high sensitivity of the convergence rate to the mesh irregularity. As an aside, we remark that the 
same study demonstrates robust convergence behavior of $P_{1}-P_{1}$ element. An explanation for the observed behavior is the very large size of velocity space, supporting features unresolved by scalar fields. Indeed, Bernard et al. (2009) partly recover the convergence rate when dissipation is introduced. Recent study by Le Roux (2012) clearly demonstrates that the lack of convergence is linked to spurious inertial oscillations maintained by this and some other discretizations.

Additional illustration in favor of this statement is offered by Danilov et al. (2008) who report difficulties with maintaining stable performance when momentum advection is not negligible. The stability is gained by computing the momentum advection in two steps. First, spatial filtering of velocity is performed by projecting it from $P_{1}^{n c}$ to $P_{1}$ representation. Second, the $P_{1}$ velocity is substituted in $(\mathbf{u} \nabla) \mathbf{u}$ at the second place. This highlights the main practical problem of this and others discretizations with too large velocity spaces - the need in tuning filtering and/or dissipation.

With this regularization the discretization shows a robust behavior. It does not support pressure modes and its velocity mass matrix is diagonal on $z$-coordinate meshes. This makes a $P_{1}^{n c}-P_{1}$ code more mathematically consistent than a $P_{1}-P_{1}$ code. However, three times larger velocity space has impact on computational efficiency, and, more importantly, the horizontal connections of $P_{1}$ scalars calls for the same compromises as in $P_{1}-P_{1}$ code. In summary, it does not lead to apparent advantages. An obvious direction here is to recast the scalar part in the FV way.

The cell-vertex discretization used by FVCOM and its large-scale implementation by Danilov (2012) have a smaller velocity space, yet it is still twice as large as in the $P_{1}$ case. With linear reconstruction upwind schemes used to advect tracer and momentum in FVCOM the code proves to be a robust performer in coastal applications. In large-scale applications on eddy resolving meshes less dissipative setups are required. This implies, in particular, other advection schemes and filtering of momentum advection in order to avoid excitation of velocity modes (Danilov (2012)). A solution that works well lies either in computing the momentum advection first on scalar control volumes and then averaging to triangles or in using the vector-invariant form. In the latter case, vorticity and energy are computed at scalar points, which provides necessary averaging. Once again, the necessity of filtering is a manifestation of unbalanced size of the velocity space.

In the end, the approach is noticeably faster than $P_{1}-P_{1}$ code. Of discretizations with practical record this one suggests, in our opinion, a good 
compromise between speed, accuracy and mathematical consistency. It, however, is rather delicate with respect to momentum dissipation, and, except for allowing for more general triangular meshes, does not offer clear advantages against hexagonal C-grid (note that their scalar parts are rather similar).

\subsection{Spherical geometry}

Discretizations using full horizontal velocities need some coordinate system, and the standard longitude-latitude representation in spherical coordinates with the north pole shifted to Greenland is the easiest option (used in FESOM). Szmelter and Smolarkiewicz (2010) show that the pole issue can be circumvented for vertex-vertex FV arrangement by special mesh design, and FVCOM employs a stereographic projection for some vicinity of geographic north pole (see Gao (2011)). More advanced technology is proposed by Comblen et al. (2009) who introduce local coordinate frames at velocity locations and on elements, and transform between them on each time step. Although this approach involves some overhead, it enables better uniformity (despite the unstructuredness, the directions of longitude-latitude coordinate axes still must vary smoothly). Note that for low-order elements triangles can be treated as locally flat, and in that case the technology of Comblen et al. (2009) can most conveniently be implemented for $P_{1}^{n c}$ and cell velocities.

For discretizations using normal velocities (C-grids) Stuhne and Peltier (2006) propose to use a Cartesian framework associated to the center of sphere. MPAS-ocean follows this approach too.

\section{Discussion}

The lack of balance between vector and scalar degrees of freedom in many proposed discretizations entails complications that are either absent or less expressed on regular quadrilateral meshes. These issues, together with the availability of accurate advection schemes and the presence of horizontal connections in CG FE vertical operators, have to be taken into account when designing 'future' unstructured-mesh codes for the large-scale ocean modeling. While the research continues, there already are solutions that work well and have a certain practical record, illustrating the utility of the concept.

Admittedly, for many discretizations stable performance is achieved through special measures which destroy their mathematical 'beauty'. We hope that examples above are sufficient to illustrate this message. This should not 
sound as warning against unstructured meshes, on the contrary, we would rather like to stress the need for a stronger feedback between practice and theory in learning about practical significance of spurious modes and the effective resolution of discretizations with differently arranged degrees of freedom.

\subsection{Discretization}

The question about the 'best' unstructured-mesh discretization for largescale ocean modeling is still under debate and calls for a dedicated comparison study. The opinions expressed in literature are as a rule based on shallowwater equations and wave dynamics, leaving all other issues unattended. In our opinion, because of hydrostatic nature of current ocean codes and the computational cost, the preference should be given to FV implementations. Among them the hexagonal C-grid (Thuburn et al. (2009), Ringler et al. (2010), see also Gassmann (2012) for a different implementation) offers a proven way to follow, and for triangular meshes, this can be the cell-vertex FV approach. It demands less sacrifice with respect to the mathematical structure than vertex-vertex discretizations (see Danilov (2012) for their comparison). Although FE codes with CG discretization for scalar fields are widely used and demonstrate robust performance in numerous practical tasks, the main objection against them is the presence of horizontal connections in vertical operators. This concerns, for example, $P_{1}-P_{1}, P_{1}^{n c}-P_{1}$ or $P_{1}^{D G}-P_{2}$ discretizations. While the latter is undoubtedly more accurate than $P_{1}-P_{1}$ pair and well suited for geostrophically dominated flows, its scalar part requires iterative solvers in a general case. It remains to see whether the resolved dynamics on $P_{1}^{D G}-P_{1}^{D G}, P_{1}^{n c}-P_{1}^{n c}$ or the balanced $B D F M_{1}-P_{1}^{D G}$ discretizations stands up for their higher computational costs. Relatedly and more generally, discontinuous FE discretizations are still insufficiently studied. Low-order representations (like $P_{1}^{D G}$ ) cluster their degrees of freedom at vertex locations. This calls for high-order methods and larger computational elements. How well such methods will behave in typical ocean applications is an open question.

The performance of these and other setups is explored fairly well on the level of shallow water equations. The important task is the intercomparison of full 3D setups, aimed at learning about their numerical efficiency, robustness in eddy-dominated regimes, spurious mixing and effective resolution in comparison with regular-mesh codes. This may help to better assess the potential of unstructured-mesh methods, and will suggest a different (from wave-motivated) metrics to judge on the utility of certain approaches. 


\subsection{Numerical efficiency}

Codes designed to work on unstructured meshes are as a rule slower than their regular-mesh counterparts per degree of freedom. This is natural to expect, and the hope is that it will be compensated by the possibility to efficiently deploy these degrees of freedom. The question, however, lies in the slowness factor. If an unstructured-mesh model is $N$ times slower per DOF, it will only be efficient against structured-mesh models if the refined area occupies $1 / N$ of the total area. In practice even this estimate may prove to be too optimistic because nesting and generalized orthogonal grids allow some flexibility in providing variable resolution on regular meshes.

Our experience with FESOM shows that it is characterized by $N$ about 10 so that it becomes competitive against regular models in tasks that require strong refinement in sufficiently small areas (like, for example, the Canadian Arctic Archipelago, Arctic Ocean, or the ice cavities around the Antarctica). Given that the refinement factor is large, the DOFs spend on representing the global ocean can be less (or even much less) in number than the DOFs in the refined region, so they are not necessarily damaging the performance. A significant part of slowness comes from 1D storage (because of consistent mass matrices) and the need for 3D neighborhood information (for tetrahedral elements).

The appearing FV codes are substantially more efficient (see, e. g., Ringler et al. (2013), Danilov (2012)). They naturally rely on the verticalhorizontal model of storage and need only the information on the horizontal neighborhood. They are characterized by $N$ about 2 to 4 , which will allow an efficient work with large refined areas. Note that with the tendency in large-scale ocean modeling to use an increased number of vertical levels (5070) the additional cost of fetching the horizontal neighborhood information becomes less and less important. What matters is the operations of reading from and writing into memory, which are generally larger in number than on structured quadrilateral meshes (for example, in both hex-C-grid and cellvertex setups the number of faces per scalar degree of freedom is larger by a factor 1.5 than on quads, so that flux contributions are written to memory more frequently). The larger count of floating-point operations is believed to become less an issue for computer architectures to come. This allows one to hope that DG codes will gain in efficiency in future, but at present they are still too slow. The view expressed here reflects our current experience.

The computational efficiency is not the only factor, and the convenience of introducing refinements in multiple regions may outweight some degree of 
slowness. Additional factors like mesh alignment with topography or coastlines or the reduced size of output may come into play too. The challenge faced by the unstructured-mesh technology as applied to large-scale ocean modeling is to propose easier to use, if somewhat slower, solutions with the multiresolution functionality.

\subsection{Advection schemes}

Although high-order advection schemes are available for FV discretization on unstructured meshes, many of them (such as the schemes proposed by Skamarock and Gassmann (2011) or Abalakin et al. (2002)) will reach their high order only if mesh is close to uniform, which has implications for the smoothness of mesh transitions. Schemes that are less sensitive to mesh non-uniformity (high-order reconstruction) are computationally more expensive, so new solutions are continuously proposed, mostly in the atmospheric community (see, i. e., recent scheme by Chen et al. (2012)) which may be of interest to ocean codes too. Many questions here still wait for their solutions. Among them are analyses of transport scheme performance in 3D cases, and the concern here is the difference in spatial resolution for vertical and horizontal velocity fields. Another issue is the impact of mesh nonuniformity and orientation. Fully unexplored are questions of spurious diapycnal mixing, especially in the context of mesh nonuniformity.

\subsection{Parameterizations and resolution in general}

Although these topics are outside the scope of this review, they need to be mentioned, since they arise in practical applications of multiresolution codes. The coefficients of horizontal viscosity and isopycnal diffusivity are commonly scaled with the cell size (to an appropriate power), but what is the optimal scaling on highly variable meshes? The Smagorinsky or Leith viscosity parameterizations contain the scaling by construction, but other parameterizations may need more care. In particular, an obvious question is how to switch on/off the eddy-induced transport parameterization of Gent and McWilliams when the mesh resolution varies from coarse to eddy-resolving. The current selection in FESOM, for example, is to vary the GM coefficient with element size, but ideally a closure is required that monitors the level of resolved eddy kinetic energy.

The question of how to apply the refinement is even more intricate. Ideally, in large-scale applications, in addition to refining the region of interest one also seeks to resolve other places known to influence the solutions, such 
as straits, or overflow sites. While including straits is straightforward, the horizontal resolution alone is insufficient to model the descent of dense water unless the vertical discretization and topography representation allow it. Practical implementations combining the $z$-coordinate with local terrain following representation, as used by Timmermann et al. (2012) for ice cavity studies, are possible, but need tuning. It is not a priori clear how wide the transitional zones should be and to what extent by locally resolving a process one gets an opportunity to correctly represent its impact on the large-scale circulation. There are many related questions, and we are only at the beginning of their analysis.

\section{Conclusions}

The unstructured-mesh models are becoming reality in large-scale ocean modeling. We believe that the understanding available now is sufficient to propose solutions that are good enough for many practical tasks. In particular, the finite-volume approaches (hex-C-grid and cell-vertex) described above can be generally recommended.

Questions on how to improve the available technology making it more efficient, accurate and easier to use still remain. The research will undoubtedly continue and may lead to new efficient approaches. However, many oceanographic questions can already be addressed with the already existing technology. In fact, even a slower method of FESOM is successfull for properly formulated problems (see, e. g., Wang et al. (2010), Wang et al. (2012), Hellmer et al. (2012), Timmermann et al. (2012), Wekerle et al. (2013)). The proposed finite-volume approaches open up new possibilities (see, e. g., Ringler et al. (2013)). In this respect it should be stressed that the feedback gained from running applications is not less important than theoretical studies. It is hoped that it will increasingly guide future development, in particular with respect to parameterizations. It is also hoped that it will improve synergy between different groups by explicitly pointing at optimal solutions.

It would be incorrect to expect that unstructured meshes will be broadly used for large-scale ocean modeling in the very nearest future. It is likewise incorrect to overlook their potential of seamless nesting for studies of ocean dynamics and regional climate in coupled systems. The task is in backing this expectation with new practical examples and easier to use solutions. 


\section{Acknowledgments}

I am indebted to my colleagues at AWI for numerous discussions and contributions. I would also like to thank S. Griffies, E. Hannert and T. Ringler for helpful comments on earlier versions of this work. Comments of anonymous reviewers are appreciated too.

\section{References}

Abalakin, I., Dervieux, A., Kozubskaya, T., 2002. A vertex-centered highorder MUSCL scheme applying to linearized Euler acoustics. INRIA, Rapport de recherche 4459.

Arakawa A., 1966. Computational design for long-term numerical integration of the equations of fluid motion: Two-dimensional incompressible flow. Part I. J. Comput. Phys., 1, 119-143.

Arakawa A., Lamb V. R., 1981, A potential enstrophy and energy conserving sheme for the shallow water equations. Mon. Wea. Rev., 109, 18-36.

Bernard, P. -E., Chevaugeon, N., Legat, V., Deleersnijder, E., Remacle, J. -F., 2007. High-order h-adaptive discontinuous Galerkin methods for ocean modelling. Ocean Dyn. 57, 109-121.

Bernard, P. -E., Deleersnijder, E. , Legat, V., Remacle, J. -F., 2008. Dispersion Analysis of Discontinuous Galerkin Schemes Applied to Poincaré, Kelvin and Rossby Waves. J. Sci. Comput. 34, 26-47.

Bernard, P.-E., Remacle, J.-F., Legat, V., 2009. Modal analysis on unstructured meshes of dispersion properties of the $P_{1}^{N C}-P_{1}$ pair. Ocean Modell. $28,2-11$.

Blain, C. A., Massey, T. C., 2005. Application of a Coupled DiscontinuousContinuous Galerkin Finite Element Shallow Water Model to Coastal Ocean Dynamics, Ocean Modell., 10, 283-315.

Blaise, S., Comblen, R., Legat, V., Remacle, J.-F., Deleersnijder, E., Lambrechts, J., 2010. A discontinuous finite element baroclinic marine model on unstructured prismatic meshes Part I: space discretization. Ocean Dyn. 60, 1371-1393. 
Blazek, J., 2001. Computational fluid dynamics: Principles and applications. Elsevier

Budgell, W. P., Oliveira, A., Skogen, M. D., 2007. Scalar advection schemes for ocean modelling on unstructured triangular grids. Ocean Dyn., 57, 339-361.

Casulli, V., Walters, R. A., 2000. An unstructured grid, three-dimensional model based on the shallow water equations. Int. J. Numer. Meth. Fluids $32,331-348$.

Chen, C., Liu, H., Beardsley, R. C., 2003. An unstructured grid, finitevolume, three-dimensional, primitive equations ocean model: Applications to coastal ocean and estuaries. J. Atmos. Ocean. Tech. 20, 159-186.

Chen, C., Bin, J., Xiao, F., 2012. A Global Multimoment Constrained FiniteVolume Scheme for Advection Transport on the Hexagonal Geodesic Grid. Mon. Wea. Rev. 140, 941-955.

Comblen, R., Legrand, S., Deleersnijder, E., Legat, V., 2009. A finite-element method for solving shallow water equations on the sphere. Ocean Modell. $28,12-23$.

Comblen, R., Blaise, S., Legat, V., Remacle, J.-F., Deleersnijder, E., Lambrechts, J., 2010. A discontinuous finite element baroclinic marine model on unstructured prismatic meshes Part II: implicit/explicit time discretization. Ocean Dyn. 60, 1395-1414.

Comblen, R., Lambrechts, J., Remacle, J.-F., Legat, V., 2010. Practical evaluation of five partly discontinuous finite element pairs for the nonconservative shallow water equations. Int. J. Numer. Meth. Fluids 63, 701724 .

Conroy, C. J., Kubatko, E. J., West, D. W., 2012. ADMESH: An advanced, automatic unstructured mesh generator for shallow water models. Ocean Dyn. DOI 10.1007/s10236-012-0574-0

Cotter, C. J., Ham, D. A., Pain, C. C., 2009. A mixed discontinuous/continuous finite element pair for shallow-water ocean modelling. Ocean Modell. 26, 86-90. 
Cotter, C. J., Ham, D. A., 2011. Numerical wave propagation for the triangular $P 1_{D G}-P 2$ finite element pair. J. Comput. Phys. 230, 2806-2820.

Cotter, C. J., Shipton, J., 2012. Mixed finite elements for numerical weather prediction. J. Comput. Phys. 231, 7076-7091.

Cotter, C. J., Thuburn, J. 2012. A finite element exterior calculus framework for the rotating shallow-water equations. arXiv:1207.3336v01.

Cueto-Felgueroso, L., Colominas, I., 2008. High-order finite-volume methods and multiresolution reproducing kernels. Arch. Comput. Methods Eng. 15, $185-228$.

Danilov, S., Kivman, G., Schröter, J., 2004. A finite element ocean model: principles and evaluation. Ocean Modell. 6, 125-150.

Danilov. S., Wang, Q., Losch, M., Sidorenko, D., Schröter, J., 2008. Modeling ocean circulation on unstructured meshes: comparison of two horizontal discretizations. Ocean Dyn. 58, 365-374.

Danilov, S., 2010. On utility of triangular C-grid type discretization for numerical modeling of large-scale ocean flows, Ocean Dyn. 60, 1361-1369.

Danilov, S., 2012. Two finite-volume unstructured mesh models for large-scale ocean modeling. Ocean Modell. 47, 14-25, doi:10.1016/j.ocemod.2012.01.004.

Dawson, C. N., Westerink, J. J., Feyen, J. C., Pothina, D., 2006. Continuous, Discontinuous and Coupled Discontinuous-Continuous Galerkin Finite Element Methods for the Shallow Water Equations. Intl. J. Num. Meth. Fluids, 52, 63-88.

Donea, J., Huerta, A., 2003. Finite element methods for flow problems. John Wiley and Sons.

Ford, R., Pain, C. C., Piggott, M. D., Goddard, A. J. H., de Oliveira, C. R. E., Umpleby, A. P., 2004. A nonhydrostatic finite-element model for threedimensional stratified oceanic flows. Part I: Model formulation, Mon. Wea. Rev., 132, 2816-2831. 
Fox-Kemper, B., Menemenlis, D., 2008. Can large eddy simulation techniques improve mesoscale rich ocean models? In: Ocean modeling in an eddying regime, Ed. M. W. Hecht and H. Hasumi, Geophysical Monograph 177, AGU, 319-337.

Fringer, O. B., Gerritsen, M., Street, R. L., 2006. An unstructured-grid, finite-volume, nonhydrostatic, parallel coastal ocean simulator. Ocean Modelling 14, 139-173.

Gao, G., 2011. An unstructured-grid finite-volume Arctic ice-ocean coupled model (AO-FVCOM): development, validation and applications. A Dissertation in Marine Science and Technology, University of Massachusetts School of Marine Sciences.

Gasmann, A., 2011. Inspection of hexagonal and triangular C-grid discretizations of the shallow water equations. J. Comput. Phys. 230, 2706-2721.

Gasmann, A., 2012. A global hexagonal C-grid non-hydrostatic dynamical core (ICON-IAP) designed for energetic consistency. Q. J. R. Meteorol. Soc. 139, 152-175. doi:10.1002/qj.1960

Greenberg, D. A., Dupont, F., Lyard, F. H., Lynch, D. R., Werner, F. E., 2007. Resolution issues in numerical models of oceanic and coastal circulation. Continental Shelf Research 27, 1317-1343.

Griffies, S. M., Böning, C., Bryan, F. O., Chassignet, E. P., Gerdes, R., Hasumi, H., Hirst, A., Treguier, A., Webb, D., 2000. Developments in ocean climate modelling, Ocean Modell. 2, 123-192.

Griffies, S. M., 2004. Fundamentals of ocean climate models. Princeton University Press.

Griffies, S. M., Biastoch, A., Böning, C., Bryan, F., Danabasoglu, G., Chassignet, E. P., England, M. H., Gerdes, R., Haak, H., Hallberg, R. W., Hazeleger, W., Jungclaus, J., Large, W. G., Madec, G., Pirani, A., Samuels, B. L., Scheinert, M., Gupta, A. S., Severijns, C. A., Simmons, H. L., Treguier, A. M., Winton, M., Yeager, S., Yin, J., 2009. Coordinated ocean-ice reference experiments (COREs). Ocean Model. 26, 1-46. doi:10.1016/j.ocemod. 2008.08.007 
Hanert, E., Le Roux, D. Y., Legat V. and Delesnijder, E., 2005 An efficient Eulerian finite element method for the shallow water equations. Ocean Modell. 10, 115-136.

Hanert, E., Legat, V., 2006. How to save a bad element with weak boundary conditions. Computers \& Fluids 35, 477-484. doi: 10.1016/j.compfluid.2005.02.005.

Hanert, E. Walters, R. A., Le Roux, D. Y., Pietrzak, J., 2009. A tale of two elements: $P_{1}^{N C}-P_{1}$ and $R T_{0}$. Ocean Modell. 28, 24-33.

Hellmer, H. H., Kauker, F., Timmermann, R., Determann, J., Rae, J., 2012. Twenty-first-century warming of a large Antarctic ice-shelf cavity by a redirected coastal current, Nature 485, 225-228. doi:10.1038/nature11064.

Hervouet, J.-M., 2000. TELEMAC modelling system: an overview. Hydrological Processes 14, 2209-2210.

Hervouet, J.-M., 2007. Hydrodynamics of Free Surface Flows: Modelling with the Finite Element Method. John Wiley and Sons.

Hua, B. L., Thomasset, F., 1984. A noise-free finite-element scheme for the two-layer shallow-water equations. Tellus 36A, 157-165.

Hundsdorfer, W., Spee, E. J., 1995. An efficient horizontal advection scheme for the modeling of global transport of constituents. Mon. Wea. Rev. 123, $3554-3564$.

Hyman J. M., Shashkov M., 1997, Natural discretizations for the divergence, gradient and curl on logically rectangular grids. Computers Math. Applic. 33, 81-104.

Kärnä, T., Legat, V., Deleersnijder. E., 2013. A baroclinic discontinuous Galerkin finite element model for coastal flows. Ocean Modell. 61, 1-20. doi:10.1016/j.ocemod.2012.09.009

Killworth, P. D., Stainforth, D., Webb, D. J., Paterson, S. M., 1991. The development of a free-surface Brian-Cox-Semptner Ocean model. J. Phys. Oceanogr. 21, 1333-1348. 
Kramer, S. C., Cotter, C. J., Pain, C. C., 2010. Solving the Poisson equation on small aspect ratio domains using unstructured meshes. Ocean Modell. 35, 253-263.

Kuzmin, D., Turek, S., 2002. Flux correction tools for finite elements. J. Comput. Phys. 175, 525-558. doi:10.1006/jcph.2001.6955

Lai, Z., Chen, C., Cowles, G. W., Beardsley, R. C., 2010. A nonhydrostatic version of FVCOM: 1. Validation experiments, J. Geophys. Res. 115, C11010, doi:10.1029/2009JC005525.

Lambrechts, J., Comblen, R., Legat, V. Geuzaine, C., Remacle, J.-F., 2008. Multiscale mesh generation on the sphere. Ocean Dyn. 58, 461-473.

Le Roux, D. Y., Staniforth, A., Lin, C. A., 1998. Finite Elements for ShallowWater Equation Ocean Models. Mon. Wea. Rev. 126, 1931-1951.

Le Roux, D. Y, 2005. Dispersion Relation Analysis of the $P_{1}^{N C}-P_{1}$ FiniteElement Pair in Shallow-Water Models. SIAM J. Sci. Comput. 27, 394-414.

Le Roux, D. Y., Rostand, V., Pouliot, B., 2007. Analysis of numerically induced oscillations in 2D finite-element shallow-water models. Part I: Inertia-gravity waves. SIAM J. Sci. Comput. 29, 331-360.

Le Roux, D. Y., Pouliot, B., 2008. Analysis of numerically induced oscillations in 2D finite-element shallow-water models. Part II: Free planetary waves, SIAM J. Sci. Comput. 30, 1971-1991.

Le Roux, D. Y., Hanert, E.,Rostand, V., Pouliot, B., 2009. Impact of mass lumping on gravity and Rossby waves in $2 \mathrm{D}$ finite-element shallow-water models. Int. J. Numer. Meth. Fluids 59, 767-790.

Le Roux, D. Y., Walters, R., Hanert, E., Pietrzak, J., 2012. A comparison of the GWCE and mixed $P 1^{N C}-P 1$ formulations in finite-element linearized shallow-water models. Int. J. Numer. Meth. Fluids 68, 1497-1523. doi:10.1002/fld.2540

Le Roux, D. Y., 2012. Spurious inertial oscillations in shallow-water models. J. Comput. Phys. 231, 7959-7987. 
Le Sommer, J., Penduff, T., Theetten, S., Madec, G., Barnier, B., 2009. How momentum advection schemes influence current-topography interactions at eddy-permitting resolution. Ocean Modell. 29, 1-14.

Li, B. Q., 2006. Discontinuous finite elements in fluid dynamics and heat transfer. Springer.

Lipscomb, W., Ringler, T., 2005. An incremental remapping transport scheme on a spherical geodesic grid. Mon. Wea. Rev. 133 2335-2350.

Löhner, R., Morgan, K., Peraire, J., Vahdati, M., 1987. Finite-element fluxcorrected transport (FEM-FCT) for the Euler and Navier-Stokes equations, Int. J. Numer. Meth. Fluids, 7, 1093-1109.

Lynch, D. R., Ip, J. T. C., Naimie, C. E., Werner, F. E., 1996. Comprehensive coastal circulation model with application to the Gulf of Maine. Cont. Shelf Res. 16, 875-906.

Marshall, J., Adcroft, A., Hill, C., Perelman, L., Heisey, C., 1997. A finitevolume, incompressible Navier-Stokes model for studies of the ocean on parallel computers. J. Geophys. Res. 102, 5753-5766.

Massey, T. C., Blain, C. A., 2006. In search of a consistent and conservative mass flux for the GWCE, Comput. Methods Appl. Mech. and Engrg. 195, 571-587.

Miura, H., 2007. An upwind-biased conservative advection scheme for spherical hexagonal-pentagonal grids. Mon. Wea. Rev. 135, 4038-4044.

Ollivier-Gooh, C., Van Altena, M., 2002. A high-order-accurate unstructured mesh finite-volume scheme for the advection/diffusion equation. J. Comput. Phys. 181, 729-752.

Ouvrard, H., Kozubskaya, T., Abalakin, I., Koobus, B., Dervieux, A., 2009. Advective vertex-centered reconstruction scheme on unstructured meshes. INRIA, Rapport de recherche 7033.

Pain, C. C., Piggott, M. D., Goddard, A. J. H., Fang, F., Gorman, G. J., Marshall, D. P., Eaton, M. D., Power, P. W., de Oliveira, C. R. E., 2005. Three-dimensional unstructured mesh ocean modelling, Ocean Modell. 10, $5-33$. 
Perot, B. 2000. Conservation properties of unstructured staggered mesh schemes. J. Comput. Phys., 159, 58-89.

Persson, P. O., Strang, G., 2004. A simple mesh generator in MATLAB, SIAM Review, 46, 329-345.

Piggott, M. D., Pain, C. C., Gorman, G. J., Marshall, D. P., Killworth, P. D., 2008. Unstructured adaptive meshes for ocean modeling. In: Ocean modeling in an eddying regime, Ed. M. W. Hecht and H. Hasumi, Geophysical Monograph 177, AGU, 383-408.

Raviart, P. A., Thomas J. M., 1977. A mixed finite element method for 2nd order elliptic problems. In Mathematical Aspects of the Finite Element Methods, Galligani I, Magenes E (eds). Lecture Notes in Mathematics. Springer: Berlin, 292-315.

Ringler, T. D., Randall, D. A., 2002. The ZM grid: an alternative to the Z grid. Mon. Wea. Rev. 130, 1411-1422.

Ringler, T., Ju, L. Gunzburger, M., 2008. A multiresolution method for climate system modeling: application of spherical centroidal Voronoi tessellations. Ocean Dynamics 58, 475-498.

Ringler, T. D., Thuburn, J., Klemp, J. B., Skamarock, W. C., 2010. A unified approach to energy conservation and potential vorticity dynamics for arbitrarily-structured C-grids. J. Comput. Phys. 229, 3065-3090.

Ringler, T., 2011. Momentum, vorticity and transport: Considerations in the design of a finite-volume dynamical core. Numerical Techniques for Global Atmospheric Models, Springer Lecture Notes in Computational Science and Engineering, Eds. P. H. Lauritzen, C. Jablonowski, M. A. Taylor and R. D. Nair.

Ringler, T., Petersen, M., Higdon, R., Jacobsen, D., Maltrud, M., Jones, P. W., 2012, A Multi-Resolution Approach to Global Ocean Modeling, accepted.

Rostand, V., Le Roux, D. Y., Carey, G., 2008. Kernel analysis of the discretized finite difference and finite element shallow-water models. SIAM J. Sci. Comput. 31, 531-556. 
Rostand, V., Le Roux, D. Y., 2008. Raviart-Thomas and Brezzi-DouglasMarini finite element approximations of the shallow-water equations. Int. J. Numer. Meth. Fluids 57, 951-976.

Sidorenko, D., Danilov, S., Wang, Q., Huerta-Casas, A., Schröter, J., 2009. On computing transports in finite-element models, Ocean Modell., 28, 6065 .

Sidorenko, D., Wang, Q., Danilov, S., Schröter, J., 2011. FESOM under Coordinated Ocean-ice Reference Experiment forcing. Ocean Dyn. 61, 881810, doi:10.1007/s10236-011-0406-7.

Skamarock, W. C., Menchaca, M., 2010. Conservative transport schemes for spherical geodesic grids: high-order reconstructions for forward-in-time schemes. Mon. Wea. Rev. 138, 4497-4508.

Skamarock, W. C., Gassmann, A., 2011. Conservative transport schemes for spherical geodesic grids: high-order flux operators for ODE-based time integration. Mon. Wea. Rev. 139, 2962-2975. doi: 10.1175/MWR-D-1005056.1.

Staniforth, A., Thuburn, J., 2011. Horizontal grids for global weather and climate prediction models: a review. Q. J. R. Meteorol. Soc. 138, 126. doi:10.1002/qj.958

Stuhne, G. R., Peltier, W. R., 2006. A robust unstructured grid discretization for 3-dimensional hydrostatic flows in spherical geometry: A new numerical structure for ocean general circulation modeling. J. Comput. Phys. 213, 704-729.

Subramanian, V., Perot, J.B., 2006. Higher-order mimetic methods for unstructured meshes. J. Comput. Phys., 219, 68-85.

Szmelter, J., Smolarkiewicz, P., 2010. An edge-based unstructured mesh discretization in geospherical framework, J. Comput. Phys. 229, 4980-4995.

Timmermann, R., Danilov, S., Schröter, J., Böning, C., Sidorenko, D., Rollenhagen, K., 2009. Ocean circulation and sea ice distribution in a finiteelement global ice-ocean model. Ocean Modell. 27, 114-129. 
Timmermann, R. , Wang, Q. and Hellmer, H., 2012. Ice shelf basal melting in a global finite-element sea ice/ice shelf/ocean model, Annals of Glaciology, $53,303-314$.

Thuburn, J. 2008. Numerical wave propagation on the hexagonal C-grid. J. Comput. Phys. 227, 5836-5858.

Thuburn, J., Ringler, T. D., Skamarock, W. C., Klemp, J. B., 2009. Numerical representation of geostrophic modes on arbitrarily structured C-grids. J. Comput. Phys. 228, 8321-8335.

Walters, R. A., Hanert, E., Pietrzak, J., Le Roux, D. Y., 2009: Comparison of unstructured, staggered grid methods for the shallow water equations, Ocean Modell., 28, 106-117.

Wan, H., Giorgetta, M.A., Zängl, G., Restelli, M., Majewski, D., Bonaventura, L., Fröhlich, K., Reinert, D., Rípodas, P., Kornblueh, L., 2013. The ICON-1.2 hydrostatic atmospheric dynamical core on triangular grids part 1: Formulation and performance of the baseline version. Geoscientific Model Development Discussions 6, 59-119.

Wang, Q., Danilov, S., Schröter, J., 2008. Finite Element Ocean circulation Model based on triangular prismatic elements, with application in studying the effect of topography representation. J. Geophys. Res. 113, C05015. doi:10.1029/2007JC004482

Wang, Q., Danilov, S., Schröter, J., 2009. Bottom water formation in the southern Weddell Sea and the influence of submarine ridges: Idealized numerical simulations. Ocean Modell. 28, 50-59.

Wang, Q., Danilov, S., Hellmer, H., Schröter, J., 2010. Overflow dynamics and bottom water formation in the western Ross Sea: The influence of tides, J. Geophys. Res. 115, C10054, doi:10.1029/2010JC006189.

Wang, X., Wang, Q., Sidorenko, D., Danilov, S. Schröter, J., Jung, T., 2012. Long-term ocean simulations in FESOM: evaluation and application in studying the impact of Greenland Ice Sheet melting. Ocean Dyn. 62, 14711486. doi: 10.1007/s10236-012-0572-2.

Webb, D.J., de Cuevas, B. A., Richmond, C., 1998. Improved advection schemes for ocean models. J. Atm. Ocean. Tech. 15, 1171-1187. 
Wekerle, C., Wang, Q., S. Danilov, J. Schröter, T. Jung, 2013. Freshwater transport through the CAA in a multi-resolution global model: Model assessment and the driving mechanism of interannual variability. J. Geoph. Res., submitted.

Westerink, J. J., Luettich, R. A., Blain, C. A., Scheffner, N. W., 1992. ADCIRC: An Advanced Three-Dimensional Circulation Model for Shelves, Coasts and Estuaries; Report 2: Users Manual for ADCIRC-2DDI. Contractors Report to the US Army Corps of Engineers. Washington D.C.

White, L., Deleersnijder, E., Legat, V., 2008a. A three-dimensional unstructured mesh shallow-water model, with application to the flows around an island and in a wind driven, elongated basin. Ocean Modell. 22, 26-47.

White, L., Legat, V., Deleersnijder, E., 2008b. Tracer conservation in a three-dimensional, finite element, free-surface, marine model on moving prismatic meshes, Mon. Wea. Rev. 136, 420-442.

Wolfram, P. J., Fringer, O. B., 2013. Mitigating horizontal divergence checker-board oscillations on unstructured triangular C-grids for nonlinear hydrostatic and nonhydrostatic flows, Ocean Modelling, submitted.

Zhang, Y.L., Baptista, A.M., Myers, E.P., 2004. A cross-scale model for 3D baroclinic circulation in estuary-plume-shelf systems: I. Formulation and skill assessment. Cont. Shelf Res. 24, 2187-2214.

Zhang, Y., Baptista, A. M., 2008. SELFE: A semi-implicit EulerianLagrangian finite-element model for cross-scale ocean circulation. Ocean Modelling 21, 71-96.

Zienkiewicz, O. C., Taylor, R. L., 2000. The finite element method. Butterworth-Heinemann, Oxford. 